\title{
A Prediction Method for Abnormal Condition of Scheduling Plan with Operation Time Delay in Steelmaking and Continuous Casting Production Process
}

\author{
Shengping $\mathrm{YU}^{*}$ \\ State Key Laboratory of Synthetical Automation for Process Industries, Northeastern University, Shenyang, Liaoning Province, \\ 110819 P. R. China.
}

(Received on July 13, 2012; accepted on March 1, 2013)

\begin{abstract}
In the steelmaking and continuous casting (SMCC) production process, the operation time delay often occurs which may lead to planned casting break or processing conflict so that the initial scheduling plan becomes unrealizable. Existing rescheduling methods with disturbances firstly classify the disturbances according to the disturbance type and disturbance quantity only by artificial experience or rules, and then directly adjust initial scheduling plan with corresponding rescheduling method. Those methods don't analyze the influence degree of disturbances to the initial scheduling plan in detail, so the adjustment degree of initial scheduling plan is always too greater, which leads to the poor continuity and stability of initial scheduling plan. In this paper, the relation among operation time delay, planned casting break and processing conflict is deeply analyzed. Then a novel prediction method for abnormal condition of scheduling plan with operation time delay disturbance in SMCC production process is proposed including disturbance identification of operating time delay based on event-driven mechanism, analysis on charges based on reachability matrix, analysis on influence degree of disturbance and abnormal condition decision of initial scheduling plan. As a result, the real-time application shows that the proposed prediction method can timely and accurately predict the abnormal condition of the scheduling plan with operation time delay disturbance in SMCC production process, which can only adjust the affected charges that must to be rescheduled in the initial scheduling plan and reduce the frequency of complete rescheduling. The initial scheduling plan can also maintain the good continuity and stability.
\end{abstract}

KEY WORDS: steelmaking and continuous casting; scheduling plan; abnormal condition; planned casting break; processing conflict; prediction.

\section{Introduction}

The SMCC production process is the core working procedure in modern large steel plant. Due to the process complexity, a large number of machines and frequent changes of the production environment, operation time delay often occurs which may lead to planned casting break or processing conflict so that the initial scheduling plan becomes unrealizable. It is of great significance to quickly and effectively adjust scheduling plan to ensure steel quality and production stability.

Nowadays the studies of rescheduling method for abnormal condition of scheduling plan with disturbances mainly focus on schedule repair and complete rescheduling according to the disturbance type and disturbance quantity. Right-shift rescheduling is adopted for smaller time delay disturbance, which means to reschedule by globally shifting the remaining operations schedule forwards in time. For the quality disturbance, equipment failure or excessive time delay, total rescheduling is used to adjust scheduling plan without consideration of the initial schedule. ${ }^{1)}$ If disturbance event is very large impact on

* Corresponding author: E-mail: spyu@mail.neu.edu.cn DOI: http://dx.doi.org/10.2355/isijinternational.53.1028 production, complete rescheduling is needed which includes production path planning and production time scheduling. ${ }^{2}$ An algorithm for rescheduling the affected operations in a job shop is presented in order to preserve as much as possible the robustness of the initial schedule, which is different from the right-shift rescheduling and complete rescheduling. ${ }^{3)}$ However, the research on scheduling method for SMCC production process focuses on static scheduling problem, ${ }^{4-6)}$ and rescheduling methods are rarely addressed. The development of a knowledge model including task, inference and domain, which describes the reasoning process in managing schedule disturbance in steel-making, is presented. ${ }^{7}$ A constraint-based approach for steelmaking-continuous casting rescheduling problem is presented for machine failure. ${ }^{8-10}$ ) A real-time scheduling method is advanced with disturbances event considering four major categories, and a rescheduling algorithm of backward method and hybrid intelligent method is proposed. ${ }^{11)}$ A general framework for using real time information to improve scheduling decisions is developed, and general measures of utility and stability are defined to evaluate strategies for deal with the deal time information. ${ }^{12)}$

Existing rescheduling methods with disturbances firstly classify the disturbances according to the disturbance type and disturbance quantity only by artificial experience or rules, and 
then directly adjust initial scheduling plan with corresponding rescheduling method. Those methods include schedule repair for part of charges, complete time rescheduling only adjusting operation time and complete rescheduling including production path planning and production time scheduling. Above methods don't analyze the influence degree of disturbances to the initial scheduling plan in detail, so the adjustment degree of initial scheduling plan is always too greater, which leads to the poor continuity and stability of initial scheduling plan.

In this paper, the disturbance type, disturbance point and disturbance value are firstly determined according to the disturbance event information. Secondly, affected charges are analyzed based on reachability matrix. Then the prediction models of abnormal condition decision of initial scheduling plan without considering buffers and with considering buffers are established to analyze influence degree of operation time delay disturbance to planned casting break and processing conflict, respectively. Finally, abnormal condition of initial scheduling plan is predicted. As a result, the real-time application shows that the proposed prediction method can timely and accurately predict the abnormal condition of the scheduling plan with operation time delay disturbance in SMCC production process, which can only adjust the affected charges in the initial scheduling plan and reduce the frequency of complete rescheduling. The initial scheduling plan can also maintain the good continuity and stability.

The paper is organized as follows. In Section 2, we describe the SMCC production process and the scheduling plan of SMCC production process. The abnormal condition of planned casting break and processing conflict is defined. Section 3 presents a novel prediction strategy for abnormal condition of scheduling plan with operation time delay disturbance in SMCC production process. In Section 4, a novel prediction method for abnormal condition of scheduling plan with operation time delay disturbance in SMCC production process is proposed, which includes disturbance identification of operating time delay based on event-driven mechanism, analysis on charges based on reachability matrix, analysis on influence degree of disturbance and abnormal condition decision of initial scheduling plan. Section 5 presents industrial application in Baosteel factory of China. Finally, conclusions are outlined in Section 6.

\section{Prediction Problem Description for Abnormal Con- dition of Scheduling Plan with Operation Time Delay Disturbance in SMCC Production Process}

\subsection{Scheduling Plan for SMCC Production Process}

Molten steel is firstly smelt in the converter. Then one ladle carries molten steel from one converter to the refining process. Molten steel in the ladle is transported to the continuous caster after refined. One ladle carrying molten steel on the processing of all processes, and the transportation of the ladle among the processes are called a Charge. Molten steel in one charge is exactly from one converter and is exactly carried by one ladle. Each charge processed on each machine is regarded as an Operation at the processing stage. The operation type and operation number of charge must meet the process requirement. All charges which are continuously drained into the same tundish in a continuous caster are called a Cast.

We denote $L_{i j}$ for the $j$ th charge of the $i$ th cast, and $i=1, \ldots$, $N, j=1, \ldots, n_{i}$, where $N$ indicates the total cast number and $n_{i}$ represents the total charge number of the $i$ th cast. Each charge $L_{i j}$ consists of $\theta_{i j}$ operations $\left(o_{i j_{1}}, \ldots, o_{i, j}, \theta_{i j}\right)$. Initial SMCC scheduling plan solution $S^{0}$ includes the initial processing machine $z_{i j k}^{0}$, the initial starting time $s_{i j k}^{0}$, the initial processing time $p_{i j k}^{0}$, the initial completion time $e_{i j k}^{0}$ of operation $o_{i j k}\left(k=1, \ldots, \theta_{i j}\right)$. In this paper, we denote $m_{g b}$ as the $b$ th machine in the gth machine group. The $h_{g}$ represents the total number of the gth machine group.

When operation time delay at time $t$ leads to casting break or operation time conflict, the original scheduling plan $S^{0}$ becomes unrealizable. It is important to analyze the influence degree of disturbances to the initial scheduling plan in detail in order to maintain the good continuity and stability.

\subsection{Operation Time Delay}

The actual operation time of charges will often deviate from the initial operation time of charges due to random factors, such as proficiency of operators, environmental parameters, etc. Such deviation is called operation time delay which includes starting time delay and completion time delay according the different time points.

\subsubsection{Starting Time Delay}

As one of raw materials for converters, molten irons of high temperature are transported by torpedo car from the blast furnace to the steelmaking plant and are poured into the ladles, and are finally poured into converters from ladles. During the transportation, molten irons need to be processed by the former slag, desulfurization, posterior slag. In those processes, the actual starting time of charges will be often later than the initial starting time because of proficiency of operators, environmental parameters, etc.

The production scheduling in SMCC production process mainly considers main equipment (converters, refining furnaces, and continuous casting machines). But in the actual production process, incoordination between the scheduling of auxiliary equipment and the scheduling of main equipment will result that crane and trolley can not be in place in time, which leads to starting time delay at refining stage.

We denote the $s_{i j k}^{*}$ for the actual starting time of operation $o_{i j k}$. If $s_{i j k}^{*}$ is later than the initial starting time $s_{i j k}^{0}$,

$$
s_{i j k}^{*}>s_{i j k}^{0}
$$

then starting time delay disturbance occurs. The $\Delta t$ indicates the time delay, and $\Delta t=s_{i j k}^{*}-s_{i j k}^{0}$.

\subsubsection{Completion Time Delay}

Due to a variety of random factors, such as the impact of machine failure, the proficiency of the operation of workers, environmental parameters, etc, it is hard to get accurate processing time and can only get an approximate data or data range for processing time of the molten steel in the equipment. In the actual production process, the processing time of charge is closely related to the molten steel temperature and components. With the changes of these factors, the actual processing time is also changing and is often different from initial processing time.

We denote the $e_{i j k}^{*}$ for the actual completion time of the operation $o_{i j k}$. If $e_{i j k}^{*}$ is later than the initial completion time $e_{i j k}^{0}$,

$$
e_{i j k}^{*}>e_{i j k}^{0}
$$

then completion time delay disturbance occurs. The $\Delta t$ indicates the time delay, and $\Delta t=e_{i j k}^{*}-e_{i j k}^{0}$.

\subsection{Classification in Abnormal Conditions of Schedul- ing Plan in SMCC Production Process}

Abnormal conditions of scheduling plan in SMCC production process mainly include planned casting break and processing conflict.

(1) Planned casting break

Casting break means that continuous caster can not pull out the slab through the mold because of a variety of reasons 
which results that continuous casting process is interrupted. In this paper, we divide casting break into "unplanned casting break" and "planned casting break". Unplanned casting break is that molten steel can not enter the mold for condensation because of nozzle clogging for low temperature of molten steel, nozzle clogging of ladle, nozzle clogging of tundish, etc. Unplanned casting break actually occurs in the production process. The following preventive measures can be adopted to reduce the number of occurrences of unplanned casting break: improving qualified rate of molten steel temperature in tundish, improving the cleanliness of molten steel, calcium treatment in molten steel, and etc. Planned casting break is that other factors affects the initial scheduling plan which results that some molten steel can not reach steel ladle turret in time according to initial scheduling plan and continuous casting is interrupted in the future because of molten steel supply interruption. Planned casting break is not what actually happened in the production process. It means that some factors, such as operation time delay, affect the initial scheduling plan, and if production is still going on according to the initial scheduling plan, then casting break will happen in the future.

If planned casting break occurs between charge $L_{i, j-1}$ and $L_{i j}$, then abnormal conditions of scheduling plan can be divided into the following four conditions:

- One-level planned casting break. It can resolve the planned casting break between charge $L_{i, j-1}$ and $L_{i j}$ only by adjusting the processing time of $L_{i, j-1}$ at continuous casting stage, or only by adjusting the processing time of $L_{i j}$ at each stage.

- Two-level planned casting break. It can not resolve the planned casting break between charge $L_{i, j-1}$ and $L_{i j}$ only by adjusting the processing time of $L_{i, j-1}$ at continuous casting stage, or only by adjusting the processing time of $L_{i j}$ at each stage. But the planned casting break between charge $L_{i, j-1}$ and $L_{i j}$ can be resolved synchronously by adjusting the processing time of $L_{i, j-1}$ at continuous casting stage and the processing time of $L_{i j}$ at each stage.

- Three-level planned casting break. It can not resolve the planned casting break between charge $L_{i, j-1}$ and $L_{i j}$ synchronously by adjusting the processing time of $L_{i, j-1}$ at continuous casting stage and the processing time of $L_{i j}$ at each stage. But the planned casting break between charge $L_{i, j-1}$ and $L_{i j}$ can be resolved synchronously by adjusting the processing time of $L_{i j_{1}}\left(j_{1} \leq j\right)$ at each stage.

- Four-level planned casting break. It can not resolve the planned casting break between charge $L_{i, j-1}$ and $L_{i j}$ synchronously by adjusting the processing time of $L_{i j_{1}}\left(j_{1} \leq j\right)$ at each stage. But the planned casting break between charge $L_{i, j-1}$ and $L_{i j}$ can be resolved synchronously by adjusting the processing machines of $L_{i j_{1}}\left(j_{1} \leq j\right)$ at steelmaking stage and at refining stage, and by adjusting the processing time of $L_{i j_{1}}$ $\left(j_{1} \leq j\right)$ at each stage.

\section{(2) Processing conflict}

SMCC process is a non-preemptive processing, which means that when one operation is being processed on the machine, other operations are prohibited to preempt the same machine. Once one operation begins to be processed, it is not allowed to interrupt until the processing finishes. If processing conflict occurs between charge $L_{i_{1} j_{1}}$ and $L_{i_{2} j_{2}}$ on machine $m_{g b}$, then abnormal conditions of processing conflict can be divided into the following five conditions:

- One-level processing conflict. It can resolve the processing conflict between charge $L_{i_{1} j_{1}}$ and $L_{i_{2} j_{2}}$ only by adjusting the processing time of $L_{i_{1} j_{1}}$ on machine $m_{g b}$, or only by adjusting the processing time of $L_{i_{2} j_{2}}$ on machine $m_{g b}$.

- Two-level processing conflict. It can not resolve the processing conflict between charge $L_{i_{1} j_{1}}$ and $L_{i_{2} j_{2}}$ only by adjusting the processing time of $L_{i_{1} j_{1}}$ on machine $m_{g b}$, or only by adjusting the processing time of $L_{i_{2} j_{2}}$ on machine $m_{g b}$. But the processing conflict between charge $L_{i_{1} j_{1}}$ and $L_{i_{2} j_{2}}$ can be resolved synchronously by adjusting the processing time of $L_{i_{1} j_{1}}$ and $L_{i_{2} j_{2}}$.

- Three-level processing conflict. It can not resolve the processing conflict between charge $L_{i_{1} j_{1}}$ and $L_{i_{2} j_{2}}$ synchronously by adjusting the processing time of $L_{i_{1} j_{1}}$ and $L_{i_{2} j_{2}}$ on machine $m_{g b}$. But the processing conflict between charge $L_{i_{1} j_{1}}$ and $L_{i_{2} j_{2}}$ can be resolved synchronously by adjusting the processing time of $L_{i_{1} j_{1}}$ and $L_{i_{2} j_{2}}$ on each stage.

- Four-level processing conflict. It can not resolve the processing conflict between charge $L_{i_{1} j_{1}}$ and $L_{i_{2} j_{2}}$ synchronously by adjusting the processing time of $L_{i_{1} j_{1}}$ and $L_{i_{2} j_{2}}$ on each stage. But the processing conflict between charge $L_{i_{1} j_{1}}$ and $L_{i_{2} j_{2}}$ can be resolved synchronously by adjusting the processing time of $L_{i_{1} j_{1}}, L_{i_{2} j_{2}}$ and other charges on each stage.

- Five-level processing conflict. It can not resolve the processing conflict between charge $L_{i_{1} j_{1}}$ and $L_{i_{2} j_{2}}$ synchronously by adjusting the processing time of $L_{i_{1} j_{1}}, L_{i_{2} j_{2}}$ and other charges on each stage. But the processing conflict between charge $L_{i_{1} j_{1}}$ and $L_{i_{2} j_{2}}$ may be resolved synchronously by adjusting the processing machines and processing time of $L_{i_{1} j_{1}}, L_{i_{2} j_{2}}$ and other charges on each stage.

\section{Prediction Strategy for Abnormal Condition of Scheduling Plan with Operation Time Delay Distur- bance in SMCC Production Process}

A four-stage prediction strategy for abnormal condition of scheduling plan with operation time delay disturbance in SMCC production process is proposed as shown in Fig. 1, which includes: disturbance identification of operating time delay based on event-driven mechanism, analysis on charges based on reachability matrix, analysis on influence degree of disturbance and abnormal condition decision of initial scheduling plan.

(1) Disturbance identification of operating time delay based on event-driven mechanism

Disturbance identification of operating time delay based on event-driven mechanism compares the actual starting time $s_{i j k}^{*}$ and the initial starting time $s_{i j k}^{0}$, or compares the actual completion time $e_{i j k}^{*}$ and the initial completion time $e_{i j k}^{0}$ according to the actual starting time $s_{i j k}^{*}$ or the actual completion time $e_{i j k}^{*}$. It can obtain the disturbance identification result according to the comparison result, which is denoted as $Y$, and $Y=\{o, m, y, \tau\}$. The $o$ is defined as the charge with operating time disturbance. The $m$ denotes the machine on which disturbance occurs. The $\tau$ indicates disturbance value. We define the $y$ as the disturbance type, $y \in$ $\left\{y_{0}, y_{1}, y_{2}, y_{3}, y_{4}\right\}$. The $y_{0}$ represents that there is no disturbance occurs. The $y_{1}$ represents that starting time delay occurs on the converter. The $y_{2}$ represents that completion time delay occurs on the converter. The $y_{3}$ represents that starting time delay occurs on the refining furnace. The $y_{4}$ represents that

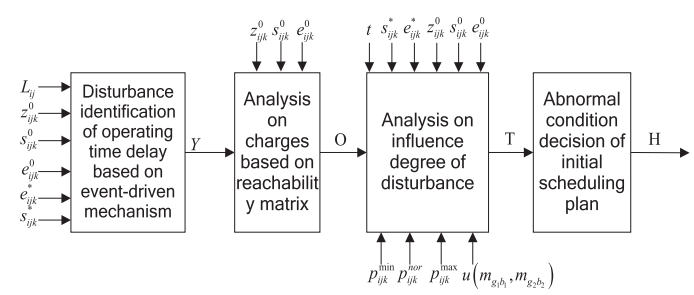

Fig. 1. Prediction strategy for abnormal condition of scheduling plan with operation time delay disturbance in SMCC production process. 
completion time delay occurs on the refining furnace.

(2) Analysis on charges based on reachability matrix

Analysis on charges based on reachability matrix is to analyze the charges which are affected by the disturbance identification result $Y$ according to the initial processing machine $z_{i j k}^{0}$, the initial starting time $s_{i j k}^{0}$ and the initial completion time $e_{i j k}^{0}$. The $\mathrm{O}$ denotes those charges set.

(3) Analysis on influence degree of disturbance

Analysis on influence degree of disturbance analyzes the influence degree of disturbance on charges from $\mathrm{O}$ according to the disturbance identification result $Y$, initial scheduling plan $S^{0}$, the minimum processing time $p_{i j k}^{\min }$, the standard processing time $p_{i j k}^{n o r}$, the maximum processing time $p_{i j k}^{\max }$, the transportation time $u\left(m_{g_{1} b_{1}}, m_{g_{2} b_{2}}\right)$ between machine $m_{g_{1} b_{1}}$ and machine $m_{g_{2} b_{2}}$. We define T as the influence degree result.

(4) Abnormal condition decision of initial scheduling plan Abnormal condition decision of initial scheduling plan predicts the abnormal condition according to the influence degree result $\mathrm{T}$. The $\mathrm{H}$ indicates the abnormal condition result, and $\mathrm{H}=\left\{m, o_{1}, O_{2}, \zeta\right\}$. The $m$ denotes the machine on which disturbance occurs. The $o_{1}$ and $o_{2}$ indicate operations with disturbance, respectively. We define the $\zeta$ as the abnormal condition type, and $\zeta \in\left\{\zeta_{11}, \zeta_{12}, \zeta_{13}, \zeta_{14}, \zeta_{21}, \zeta_{22}, \zeta_{23}, \zeta_{24}, \zeta_{25}\right\}$. The $\zeta_{11}, \zeta_{12}$, $\zeta_{13}$ and $\zeta_{14}$ denote one-level planned casting break, two-level planned casting break, three-level planned casting break and four-level planned casting break, respectively. The $\zeta_{21}, \zeta_{22}$, $\zeta_{23}, \zeta_{24}$ and $\zeta_{25}$ represent one-level processing conflict, twolevel processing conflict, three-level processing conflict, four-level processing conflict and five-level processing conflict, respectively.

\section{A Novel Prediction Method for Abnormal Condition of Scheduling Plan with Operation Time Delay Dis- turbance in SMCC Production Process}

\subsection{Disturbance Identification of Operating Time Delay Based on Event-driven Mechanism}

In the SMCC production process, once the operation is being processed or finished, dynamic event information collected from actual production includes the machine code $m$, operation $o$, processing status type $\chi$, occurrence time of processing status $t$. If processing status is starting, then $\chi=$ 1. If processing status has been finished, then $\chi=2$. Disturbance identification model is established according above dynamic event information as follows:

$$
Y=f\left(m, o, \chi, t, s_{i j k}^{0}, e_{i j k}^{0} \mid o_{i j k} \in \mathrm{O}\right)
$$

where $\mathrm{O}$ denotes the unfinished operations set. The $s_{i j k}^{0}$ and $e_{i j k}^{0}$ indicate the initial starting time and the initial completion time of operation $o_{i j k}$ from $\mathrm{O}$, respectively. If operation $o_{i j k}$ is not processed, then $\delta_{i j k}^{1}=0$. If operation $o_{i j k}$ is being processed, then $\delta_{i j k}^{1}=1$. If operation $o_{i j k}$ has not finished being processed, then $\delta_{i j k}^{2}=0$. If operation $o_{i j k}$ has been processed, then $\delta_{i j k}^{2}=1$. The disturbance identification algorithm of operating time delay based on event-driven mechanism is described in detail as follows:

Step1: Initialize the scheduling information: $S^{0}=\left\{z_{i j k}^{0}, s_{i j k}^{0}\right.$, $\left.e_{i j k}^{0} \mid o_{i j k} \in L_{i j}, L_{i j} \in \Omega\right\}$, where $\Omega$ is the set of unfinished casting charges.

Step2: Initialize the operations processing status: $\forall o_{i j k} \in \mathrm{O}$, $\delta_{i j k}^{1}=0, \delta_{i j k}^{2}=0$.

Step3: If event occurs, then get event information: machine code $m$, operation $o$, processing status type $\chi$, occurrence time of processing status $t$. Select the operation $o_{i_{1} j_{1} k_{1}}$ from $\mathrm{O}$ which $o_{i_{1} j_{1} k_{1}}$ is equal to operation $o$. If $\chi=1$, then go to the next step, else go to Step5.

Step4: Let $\delta_{i_{1} j_{1} k_{1}}^{1}=1, s_{i_{1} j_{1} k_{1}}^{*}=t, \tau=s_{i_{1} j_{1} k_{1}}^{*}-s_{i_{1} j_{1} k_{1}}^{0}$. If $\tau=0$, then $y=y_{0}$. If $k=1, \tau>0$, then $y=y_{1}$. If $1<k<\theta_{i j}, \tau>0$ then $y=y_{3}$.

Step5: Let $\delta_{i_{1} j_{1} k_{1}}^{2}=1, e_{i_{1} j_{1} k_{1}}^{*}=t, \tau=e_{i_{1} j_{1} k_{1}}^{*}-e_{i_{1} j_{1} k_{1}}^{0}$. If $\tau=0$, then $y=y_{0}$. If $k=1, \tau>0$, then $y=y_{2}$. If $1<k<\theta_{i j}, \tau>0$, then $y=y_{4}$.

Step6: Get the final disturbance identification result $Y$, and $Y=\{o, m, y, \tau\}$.

\subsection{Analysis on Charges Based on Reachability Matrix}

In the SMCC production, it must have been processed on the former operation $o_{i j k}$ and then it can be processed on the next operation $o_{i, j, k+1}$ for each charge. The adjacent charges in the same machine must be processed one by one. So, when operation time delay of one operation occurs, it will affects other operations and scheduling performance, even make the initial scheduling plan become unrealizable. It firstly needs to analyze the charges which are affected by the disturbance identification in order to analyze the influence degree of disturbances to the initial scheduling plan $S^{0}$. The working status of each machine is divided into idle, processing the first operation or middle operation of one charge and processing the last operation of one charge. We define the $\square, \bigcirc, \triangle$ as those three status, respectively. The interval time between adjacent operations from the same charge is the sum of the transportation time and waiting time, which is indicated as ellipse. The scheduling plan including two charges is as shown in Fig. 2(a). $L_{11}=\left\{o_{111}, o_{112}, o_{113}\right\}$ and $L_{12}=\left\{o_{121}, o_{122}, o_{123}\right\}$. Operations of each charge are processed by $1 \mathrm{LD}, 1 \mathrm{RH}$ and $1 \mathrm{CC}$. The time constraints relationship between operations of charge $L_{11}$ and $L_{12}$ is shown in Fig. 2(b). The node 2 and node 4 denote operation $o_{111}$ and operation $o_{121}$ on machine 1LD, respectively. The node 8 and node 10 denote operation $o_{112}$ and operation $o_{122}$ on machine $1 \mathrm{RH}$, respectively. The node 14 and node 15 denote operation $o_{113}$ and operation $o_{123}$ on machine $1 \mathrm{CC}$, respectively. The node 5 denotes the interval time between operation $o_{111}$ and operation $o_{112}$. The node 6 denotes the interval time between operation $o_{121}$ and operation $o_{122}$. The node 11 and node 12 denote the interval time between operation $o_{112}$ and operation $o_{113}$ and the interval time between operation $o_{122}$ and operation $o_{123}$, respectively. The nodes 1, 3, 7, 9, 13 indicate the idle time.

The arrows between operations indicate the influence direction of operating time delay as shown in Fig. 2(b). For example, the arrows between operations on the same machine represent that the operating time delay of former operation will cause the operating time delay of the subsequent other operation. The arrows between operations on the different machine denote that the operating time delay of former operation will cause the operating time delay of the subsequent operation from the same charge.

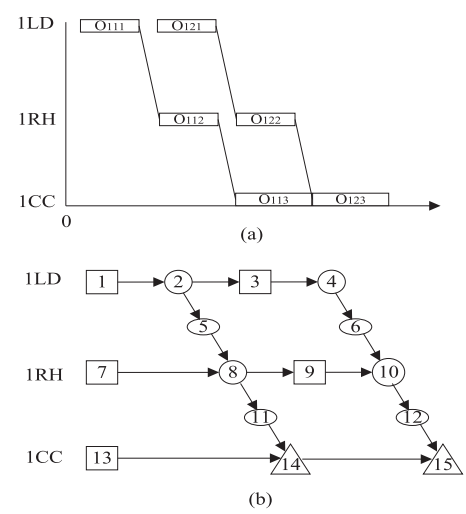

Fig. 2. The time constraints relationship between operations of charges. 
The reachability matrix $R$, which reflects the system node connectivity in graph theory, is introduced to represent the interaction relationship between operations in order to analyze the influence degree of disturbances to the initial scheduling plan $S^{0}$. $R$ can be obtained by the adjacency matrix $C$ which indicates the adjacency relationship of nodes. The adjacency matrix $C$ is defined as follows:

$$
C=\left\|c_{l_{1} l_{2}}\right\| \quad l_{1}, l_{2}=1, \ldots, N
$$

where $l_{1}$ and $l_{2}$ denote the nodes, respectively. $N$ indicates the total number of nodes. If node $l_{1}$ and node $l_{2}$ are adjacent, and the arrow points to the node $l_{2}$ from the node $l_{1}$, then $c_{l_{1} l_{2}}=1$, else $c_{l_{1} l_{2}}=0$. For example, the adjacency matrix $C$ in Fig. 2(b) is as follows:

$C=\left[\begin{array}{cccccccccccccccc} & 1 & 2 & 3 & 4 & 5 & 6 & 7 & 8 & 9 & 10 & 11 & 12 & 13 & 14 & 15 \\ 1 & 0 & 1 & 0 & 0 & 0 & 0 & 0 & 0 & 0 & 0 & 0 & 0 & 0 & 0 & 0 \\ 2 & 0 & 0 & 1 & 0 & 1 & 0 & 0 & 0 & 0 & 0 & 0 & 0 & 0 & 0 & 0 \\ 3 & 0 & 0 & 0 & 1 & 0 & 0 & 0 & 0 & 0 & 0 & 0 & 0 & 0 & 0 & 0 \\ 4 & 0 & 0 & 0 & 0 & 0 & 1 & 0 & 0 & 0 & 0 & 0 & 0 & 0 & 0 & 0 \\ 5 & 0 & 0 & 0 & 0 & 0 & 0 & 0 & 1 & 0 & 0 & 0 & 0 & 0 & 0 & 0 \\ 6 & 0 & 0 & 0 & 0 & 0 & 0 & 0 & 0 & 0 & 1 & 0 & 0 & 0 & 0 & 0 \\ 7 & 0 & 0 & 0 & 0 & 0 & 0 & 0 & 1 & 0 & 0 & 0 & 0 & 0 & 0 & 0 \\ 8 & 0 & 0 & 0 & 0 & 0 & 0 & 0 & 0 & 1 & 0 & 1 & 0 & 0 & 0 & 0 \\ 9 & 0 & 0 & 0 & 0 & 0 & 0 & 0 & 0 & 0 & 1 & 0 & 0 & 0 & 0 & 0 \\ 10 & 0 & 0 & 0 & 0 & 0 & 0 & 0 & 0 & 0 & 0 & 0 & 1 & 0 & 0 & 0 \\ 11 & 0 & 0 & 0 & 0 & 0 & 0 & 0 & 0 & 0 & 0 & 0 & 0 & 0 & 1 & 0 \\ 12 & 0 & 0 & 0 & 0 & 0 & 0 & 0 & 0 & 0 & 0 & 0 & 0 & 0 & 0 & 1 \\ 13 & 0 & 0 & 0 & 0 & 0 & 0 & 0 & 0 & 0 & 0 & 0 & 0 & 0 & 1 & 0 \\ 14 & 0 & 0 & 0 & 0 & 0 & 0 & 0 & 0 & 0 & 0 & 0 & 0 & 0 & 0 & 1 \\ 15 & 0 & 0 & 0 & 0 & 0 & 0 & 0 & 0 & 0 & 0 & 0 & 0 & 0 & 0 & 0\end{array}\right]$

The reachability matrix $R$ can be obtained by the adjacency matrix $C$ as follows:

$$
R=\left\|r_{l_{1} l_{2}}\right\| \quad l_{1}, l_{2}=1, \ldots, N
$$

If there exists directed path from the node $l_{1}$ to the node $l_{2}$, then $r_{l_{1} l_{2}}=1$, else $r_{l_{1} l_{2}}=0$. When one node $l$ task represented by $\bigcirc$ in Fig. 2(b) delays, it will only make other node task delay which is connected with the node $l$ and does not make other node task delay which is not connected with the node $l$. It can clearly identify the operations which are affected by operation time delay by the reachability matrix. The reachability matrix in Fig. 2(b) is as follows:

$$
R=\left[\begin{array}{cccccccccccccccc} 
& 1 & 2 & 3 & 4 & 5 & 6 & 7 & 8 & 9 & 10 & 11 & 12 & 13 & 14 & 15 \\
1 & 1 & 1 & 1 & 1 & 1 & 1 & 0 & 1 & 1 & 1 & 1 & 1 & 0 & 1 & 1 \\
2 & 0 & 1 & 1 & 1 & 1 & 1 & 0 & 1 & 1 & 1 & 1 & 1 & 0 & 1 & 1 \\
3 & 0 & 0 & 1 & 1 & 0 & 1 & 0 & 0 & 0 & 1 & 0 & 1 & 0 & 0 & 1 \\
4 & 0 & 0 & 0 & 1 & 0 & 1 & 0 & 0 & 0 & 1 & 0 & 0 & 0 & 0 & 1 \\
5 & 0 & 0 & 0 & 0 & 1 & 0 & 0 & 1 & 1 & 1 & 1 & 1 & 0 & 1 & 1 \\
6 & 0 & 0 & 0 & 0 & 0 & 1 & 0 & 0 & 0 & 1 & 0 & 1 & 0 & 0 & 1 \\
7 & 0 & 0 & 0 & 0 & 0 & 0 & 1 & 1 & 1 & 1 & 1 & 1 & 0 & 1 & 1 \\
8 & 0 & 0 & 0 & 0 & 0 & 0 & 0 & 1 & 1 & 1 & 1 & 1 & 0 & 1 & 1 \\
9 & 0 & 0 & 0 & 0 & 0 & 0 & 0 & 0 & 1 & 1 & 0 & 1 & 0 & 0 & 1 \\
10 & 0 & 0 & 0 & 0 & 0 & 0 & 0 & 0 & 0 & 1 & 0 & 1 & 0 & 0 & 1 \\
11 & 0 & 0 & 0 & 0 & 0 & 0 & 0 & 0 & 0 & 0 & 1 & 0 & 0 & 1 & 1 \\
12 & 0 & 0 & 0 & 0 & 0 & 0 & 0 & 0 & 0 & 0 & 0 & 1 & 0 & 0 & 1 \\
13 & 0 & 0 & 0 & 0 & 0 & 0 & 0 & 0 & 0 & 0 & 0 & 0 & 1 & 1 & 1 \\
14 & 0 & 0 & 0 & 0 & 0 & 0 & 0 & 0 & 0 & 0 & 0 & 0 & 0 & 1 & 1 \\
15 & 0 & 0 & 0 & 0 & 0 & 0 & 0 & 0 & 0 & 0 & 0 & 0 & 0 & 0 & 1
\end{array}\right]
$$

\subsection{Analysis on Influence Degree of Disturbance}

4.3.1. Analysis on Processing Conflict Caused by Operation Time Delay

It can find all operations which are affected by operation time delay by the reachability matrix. When node $l$ delays $\Delta t$ minutes, then the node which is connected with the node $l$ will also delay $\Delta t$ minutes.

When the starting time of the node $l_{0}$ denoting the operation $o_{i j k_{1}}$ delays $\Delta t$ minutes, that is $\Delta t=s_{i j k_{1}}^{*}-s_{i j k_{1}}^{0}$, recalculate the starting time $s_{i j k_{1}}$ and the completion time $e_{i j k_{1}}$ of the operation $o_{i j k_{1}}$ as follows:

$$
\begin{gathered}
s_{i j k_{1}}=s_{i j k_{1}}^{*}=s_{i j k_{1}}^{0}+\Delta t \ldots \\
e_{i j k_{1}}=s_{i j k_{1}}+p_{i j k_{1}}^{0}=e_{i j k_{1}}^{0}+\Delta t
\end{gathered}
$$

where $p_{i j k_{1}}^{0}$ is the processing time of operation $o_{i j k_{1}}$ in the initial scheduling plan. When the completion time of the node $l_{0}$ delays $\Delta t$ minutes, that is $\Delta t=e_{i j k_{1}}^{*}-e_{i j k_{1}}^{0}$, recalculate the starting time $s_{i j k_{1}}$ and the completion time $e_{i j k_{1}}$ of the operation $o_{i j k_{1}}$ as follows:

$$
\begin{gathered}
s_{i j k_{1}}=s_{i j k_{1}}^{0} \cdots . \\
e_{i j k_{1}}=e_{i j k_{1}}^{0}+\Delta t
\end{gathered}
$$

The new starting time $s_{i j k}$ and the new completion time $e_{i j k}$ of the subsequent operations of $o_{i j k_{1}}$ are recalculated as follows:

$$
\begin{array}{r}
s_{i j k}=e_{i, j, k-1}+u\left(z_{i, j, k-1}^{0}, z_{i j k}^{0}\right) \quad k=k_{1}+1, \ldots, \theta_{i j} . . \\
e_{i j k}=s_{i j k}+p_{i j k}^{0} \quad k=k_{1}+1, \ldots, \theta_{i j} \ldots \ldots \ldots \ldots . .
\end{array}
$$

where $z_{i, j, k-1}^{0}$ and $z_{i j k}^{0}$ are the processing machine of the operation $o_{i, j, k-1}$ and the processing machine of the operation $o_{i j k}$, respectively. The $u\left(z_{i, j, k-1}^{0}, z_{i j k}^{0}\right)$ is the standard transportation time between $o_{i, j, k-1}$ and $o_{i j k}$. We define the $o_{I(i j k)}$ as the subsequent operation of the operation $o_{i j k}$ on machine $z_{i j k}^{0}$. The $\chi_{I(i j k)}^{i j k}$ denotes the processing conflict time as follows:

$$
\chi_{I(i j k)}^{i j k}=\min \left(e_{i j k}, e_{I(i j k)}^{0}\right)-\max \left(s_{i j k}, s_{I(i j k)}^{0}\right) \quad k=k_{1}, \ldots, \theta_{i j}
$$

The formula (14) can be rewritten as follows according to the formula (8) to formula (13):

$$
\begin{gathered}
\chi_{I(i j k)}^{i j k}=\min \left(e_{i j k_{1}}+\sum_{k_{2}=k_{1}+1}^{k} p_{i j k_{2}}^{0}+\sum_{k_{3}=k_{1}}^{k-1} u\left(z_{i j k_{3}}^{0}, z_{i, j, k_{3}+1}^{0}\right), e_{I(i j k)}^{0}\right) \\
-\max \left(s_{i j k_{1}}+\sum_{k_{2}=k_{1}}^{k-1} p_{i j k_{2}}^{0}+\sum_{k_{3}=k_{1}}^{k-1} u\left(z_{i j k_{3}}^{0}, z_{i, j, k_{3}+1}^{0}\right), s_{I(i j k)}^{0}\right) \\
k=k_{1}, \ldots, \theta_{i j}
\end{gathered}
$$

The formula (15) is the relational model between the processing conflict time between operation $o_{i j k}$ and other operation $o_{i j k}\left(k=k_{1}, \ldots, \theta_{i j}\right)$ and the operation delay time $\Delta t$ of operation $o_{i j k_{1}}$ when the operation delay time of $o_{i j k_{1}}$ is $\Delta t$. If $\chi_{I(i j k)}^{i j k} \leq 0$, then the processing conflict does not occur. If $\chi_{I(j i k)}^{i j k}>0$, then the processing conflict occurs and the conflict time is $\chi_{I(i j k)}^{i j k}$.

\subsubsection{Analysis on Planned Casting Break Caused by} Operation Time Delay

When the delay time of operation $o_{i j k_{1}}$ is $\Delta t$, then the new starting time of $o_{i, j}, \theta_{i j}$ can be recalculated as follows according to the formula (8) to formula (13):

$$
s_{i, j, \theta_{i j}}=e_{i j k_{1}}^{0}+\Delta t+\sum_{k_{2}=k_{1}+1}^{\theta_{i j}-1} p_{i j k_{2}}^{0}+\sum_{k_{3}=k_{1}}^{\theta_{i j}-1} u\left(z_{i j k_{3}}^{0}, z_{i, j, k_{3}+1}^{0}\right)
$$


We define $\gamma_{j-1, j}^{i}$ as the planned casting break time between charge $L_{i, j-1}$ and charge $L_{i j}$ :

$$
\gamma_{j-1, j}^{i}=s_{i, j, \theta_{i j}}-e_{i, j-1, \theta_{i, j-1}}^{0}
$$

The formula (17) can be rewritten as follows according to the formula (16):

$$
\gamma_{j-1, j}^{i}=e_{i j k_{1}}^{0}+\Delta t+\sum_{k_{2}=k_{1}+1}^{\theta_{i j}-1} p_{i j k_{2}}^{0}+\sum_{k_{3}=k_{1}}^{\theta_{i j}-1} u\left(z_{i j k_{3}}^{0}, z_{i, j, k_{3}+1}^{0}\right)-e_{i, j-1, \theta_{i, j-1}}^{0} \ldots . .
$$

The formula (18) is the relational model between the planned casting break time and the operation delay time $\Delta t$ of operation $o_{i j k_{1}}$ when the operation delay time of $o_{i j k_{1}}$ is $\Delta t$. If $\gamma_{j-1, j}^{i} \leq 0$, then the planned casting break does not occur. If $\gamma_{j-1, j}^{i}>0$, then the planned casting break occurs and the planned casting break time is $\gamma_{j-1, j}^{i}$.

\subsubsection{Buffer Units of Scheduling System in SMCC Pro- duction Process}

When the operation delay time of $o_{i j k_{1}}$ is $\Delta t$, we can obtain the processing conflict time and the planned casting break time according to the formula (15) and (18). If the node $l_{0}$ has the buffer function, then the operation time delay of the subsequent connected node can be reduced through the buffer function. Supposing that the buffer time of the node $l_{0}$ is $T$, then the operation time delay of the subsequent connected node is $\Delta t-T$. If $\Delta t-T<0$, then the operation time of the subsequent connected node will not delay. When the operation delay time of node $l_{1}$ is $\Delta t$, the buffer units of scheduling system in SMCC production process are as follows:

(1) The idle time on machines. If the node $l_{1}$ connects to the node of the idle time, then the node of the idle time will play the role of time compensation. Supposing that the idle time is $T$, then the operation time delay of the subsequent connected node is $\Delta t-T$. If $\Delta t-T<0$, then the operation time of the subsequent connected node will not delay.

(2) Adjustment range of processing time of charge on the machine. We can only get a range of processing time of operations because the processing time of operations is affected by randomness. The processing time includes the minimum processing time $p_{i j k}^{\min }$, the standard processing time $p_{i j k}^{n o r}$, the maximum processing time $p_{i j k}^{\max }$. The processing time of operation $o_{i j k}$ can be adjusted in $\left[p_{i j k}^{\min }, p_{i j k}^{\max }\right]$. Supposing that the processing time of $o_{i j k}$ in the initial scheduling plan is $p_{i j k}^{0}$. If $\Delta t-\left(p_{i j k}^{0}-p_{i j k}^{\min }\right)<0$, then the operation time of the subsequent connected node will not delay.

(3) The interval time between adjacent operations of the same charge. SMCC process is a non-preemptive processing, which means that when one operation is being processed on the machine, other operations are prohibited to preempt the same machine. Once one operation begins to be processed, it is not allowed to interrupt until the processing finishes. If the interval time between adjacent operations of the same charge includes the waiting time, then it has the buffer function. Supposing that the interval time between $o_{i j k}$ and $o_{i, j, k+1}$ is $u_{i j k}^{0}\left(z_{i j k}^{0}, z_{i, j, k+1}^{0}\right)$. If $\Delta t-\left(u^{0}\left(z_{i j k}^{0}, z_{i, j, k+1}^{0}\right)-u\left(z_{i j k}^{0}, z_{i, j, k+1}^{0}\right)\right)<0$, then the operation time of the subsequent connected node will not delay.

The buffer matrix is defined as follows according to the above analysis:

$$
\mathrm{E}=\left\|\varepsilon_{l}\right\| \quad l=1, \ldots, N
$$

where $\varepsilon_{l}$ denotes the buffer time of the node $l$.

(1) If the node $l$ is the node of the idle time $T$, then

$$
\varepsilon_{l}=T
$$

(2) If the node $l$ indicates the operation $o_{i j k}$ of the charge, then

$$
\varepsilon_{l}=p_{i j k}^{0}-p_{i j k}^{\min }
$$

(3) If the node $l$ denotes the interval time between adjacent operations of the same charge, then

$$
\varepsilon_{l}=u^{0}\left(z_{i j k}^{0}, z_{i, j, k+1}^{0}\right)-u\left(z_{i j k}^{0}, z_{i, j, k+1}^{0}\right)
$$

where $u^{0}\left(z_{i j k}^{0}, z_{i, j, k+1}^{0}\right)$ is the interval time between $o_{i j k}$ and $o_{i, j, k+1}$, and $u\left(z_{i j k}^{0}, z_{i, j, k+1}^{0}\right)$ is the standard transportation time between $o_{i j k}$ and $o_{i, j, k+1}$.

If $\varepsilon_{l}>0$ in the buffer matrix E, it means that the node $l$ has the buffer time. The operation time delay of the subsequent connected node can be reduced through the buffer time. If the operation delay time of the node $l$ is $\Delta t$, then the operation time delay of the subsequent connected node can be reduced to $\Delta t-\varepsilon_{l}$. If $\varepsilon_{l}=0$, then the operation time delay of the subsequent connected node will also be $\Delta t$.

It can be known that we can obtain the processing conflict time and the planned casting break time according to the formula (15) and (18) when the operation delay time of $o_{i j k_{1}}$ is $\Delta t$. If there have buffer units, then the processing conflict time and the planned casting break time can not be directly obtained according to the formula (15) and formula (18). These are closely related with the buffer units. So, it is needed to deeply analyze the relationship between delay time $\Delta t$ and buffer units in order to find out the really impact on the scheduling system by delay time $\Delta t$.

\subsubsection{Analysis on Influence Degree of Disturbance by} Operation Time Delay under Buffer Units

When the operation delay time of $o_{i j k_{1}}$ is $\Delta t$, the analysis model of the impact on the starting time of operations by operation time delay under buffer units is established as follows according to the reachability matrix $R$ and the buffer matrix $E$ :

$$
\mathrm{T}_{1}\left(l_{0}, \Delta t\right)=\left\|\eta_{l}\right\| \quad l=1, \ldots, N
$$

where the $\eta_{l}$ denotes the final delay time of the starting time of the node $l$ affected by the delay time $\Delta t$ of the node $l_{0}$. If $r_{l_{0} l}=0$, then $\eta_{l}=0$. If $r_{l_{0} l}=1$ and there exists $B(B \geq 1)$ directed paths from the node $l_{0}$ to the node $l$, then the $\eta_{l}$ is equal to the maximum delay time of the starting time of all nodes in the directed paths:

$$
\eta_{l}=\max _{b=1, \ldots, B}\left(\Delta t-\sum_{\substack{l_{1} \in P_{b}\left(l_{0}, l\right) \\ l_{1} \neq l}} \sum_{l_{2} \in P_{b}\left(l_{0}, l\right) \backslash} c_{l_{1} \neq l} \varepsilon_{l_{1}}\right) \quad r_{l_{0} l}=1 \ldots \ldots .
$$

where $P_{b}\left(l_{0}, l\right)$ is the bth directed path in $B$ directed paths.

The analysis model of the impact on the completion time of operations by operation time delay under buffer units is established as follows according to the formula (19) and the formula (23):

$$
\mathrm{T}_{2}\left(l_{0}, \Delta t\right)=\left\|\lambda_{l}\right\| \quad l=1, \ldots, N
$$

where the $\lambda_{l}$ indicates the final delay time of the completion time of the node $l$ affected by the delay time $\Delta t$ of the node $l_{0}$. If $r_{l_{0}}=0$, then $\lambda_{l}=0$. If $r_{l_{0} l}=1$, then $\lambda_{l}=\eta_{l}-\varepsilon_{l}$.

According to the above analysis, the analysis model of the processing conflict time between adjacent operations is established as follows when the operation delay time is $\Delta t$ :

$$
\tilde{\chi}_{I(i j k)}^{i j k}=\min \left(e_{i j k}^{0}+\lambda_{l_{1}}, e_{I(i j k)}^{0}+\lambda_{l_{2}}\right)-\max \left(s_{i j k}^{0}+\eta_{l_{1}}, s_{I(i j k)}^{0}+\eta_{l_{2}}\right) \ldots
$$

where the $o_{i j k_{1}}$ is the corresponding operation for the node $l_{0}$, the $o_{i j k}$ is the corresponding operation for the node $l_{1}$ and the $o_{I(i j k)}$ is the corresponding operation for the node $l_{2}$. If $\tilde{\chi}_{I(j k)}^{i j k} \leq 0$, then there is no processing conflict time between $o_{i j k}$ and $o_{I(i j k)}$ through the buffer units. If $\tilde{\chi}_{I(i j k)}^{i j k}>0$, it means that there is still processing conflict time between $o_{i j k}$ and 
$o_{I(i j k)}$ through the buffer units.

The analysis model of the planned casting break time between adjacent charges in the same cast is established as follows when the operation delay time is $\Delta t$ :

$$
\tilde{\gamma}_{j-1, j}^{i}=\left(s_{i, j, \theta_{i j}}^{0}+\eta_{l_{2}}\right)-\left(e_{i, j-1, \theta_{i, j-1}}^{0}+\lambda_{l_{1}}\right)=\eta_{l_{2}}-\lambda_{l_{1}}
$$

where the $o_{i, j, \theta_{i j}}$ is the corresponding operation for the node $l_{2}$ and the $o_{i, j-1, \theta_{i, j-1}}$ is the corresponding operation for the node $l_{1}$. If $\tilde{\gamma}_{j-1, j}^{i} \leq 0$, there is no planned casting break time between $o_{i, j-1, \theta_{i, j-1}}$ and $o_{i, j, \theta_{i j}}$ through the buffer units. If $\tilde{\gamma}_{j-1, j}^{i}>0$, there is still planned casting break time between $o_{i, j-1, \theta_{i, j-1}}$ and $o_{i, j, \theta_{i j}}$ through the buffer units.

\subsection{Abnormal Condition Decision of Initial Scheduling Plan}

The formula (26) and the formula (27) denote the final the processing conflict time and the final planned casting break time under buffer units, respectively. The processing conflict time and the planned casting break time are resolved by under buffer units in fact, which is the adjustment method. The different abnormal condition of the scheduling plan by the operation time delay can lead to the different adjustment method to resolve the abnormal condition. So, prediction for abnormal condition of scheduling plan with operation time delay disturbance in SMCC production process is very important.

4.4.1. The Planned Casting Break Decision of Initial Scheduling Plan by Operation Time Delay under Buffer Units

The $G$ denotes the time constraint graph between operations. The $V$ indicates the all nodes set in $G$. When the node $l_{0}\left(l_{0}\right.$ is correspond to the charge $\left.L_{i j}\right)$ delay $\Delta t$, if $\gamma_{j-1, j}^{i}>0$, then there is planned casting break time between $L_{i, j-1}$ and $L_{i j}$. The $o_{i, j-l, \theta_{i, j-1}}$ is the corresponding operation for the node $l_{1}$ and the $o_{i, j, \theta_{i j}}$ is the corresponding operation for the node $l_{2}$. We define the $V_{i j}$ as the all nodes set for charge $L_{i j}$. The planned casting break decision is made as follows:

(1) If $p_{i, j-1, \theta_{i, j-1}}^{\max }-p_{i, j-1, \theta_{i, j-1}}^{0}-\gamma_{j-1, j}^{i} \geq 0$, then the abnormal condition of scheduling plan is one-level planned casting break $\zeta_{11}$.

Proof. When the node $l_{0}\left(l_{0}\right.$ is correspond to operation $o_{i j k_{1}}$ the charge $L_{i j}$ ) delay $\Delta t$, according to the formula (18),

$$
\gamma_{j-1, j}^{i}=e_{i j k_{1}}^{0}+\Delta t+\sum_{k_{2}=k_{1}+1}^{\theta_{i j}-1} p_{i j k_{2}}^{0}+\sum_{k_{3}=k_{1}}^{\theta_{i j}-1} u\left(z_{i j k_{3}}^{0}, z_{i, j, k_{3}+1}^{0}\right)-e_{i, j-1, \theta_{i, j-1}}^{0} . e_{i, j-1, \theta_{i, j-1}}^{0} \text { is }
$$

the initial completion time of operation $o_{i, j-1, \theta_{i, j-1}}$ and the initial processing time of operation $o_{i, j-1, \theta_{i, j-1}}$ is $p_{i, j-1, \theta_{i, j-1}}^{0}$. If the processing time of operation $o_{i, j-1, \theta_{i, j-1}}$ is adjusted to $p_{i, j-1, \theta_{i, j-1}}^{\max }$, then the new completion time $e_{i, j-1, \theta_{i, j-1}}$ of operation $o_{i, j-1, \theta_{i, j-1} \text { can }}$ be recalculated as follows: $e_{i, j-1, \theta_{i, j-1}}=e_{i, j-1, \theta_{i, j-1}}^{0}+p_{i, j-1, \theta_{i, j-1}}-$ $p_{i, j-1, \theta_{i, j-1}}^{0}$. Then according to the formula (18), the new planned casting break time (denoted as $\left.\left(\gamma_{j-1, j}^{i}\right)^{\prime}\right)$ can be recalculated as follows:

$$
\begin{aligned}
\left(\gamma_{j-1, j}^{i}\right)^{\prime} & =e_{i j k_{1}}^{0}+\Delta t+\sum_{k_{2}=k_{1}+1}^{\theta_{i j}-1} p_{i j k_{2}}^{0}+\sum_{k_{3}=k_{1}}^{\theta_{i j}-1} u\left(z_{i j k_{3}}^{0}, z_{i, j, k_{3}+1}^{0}\right)-e_{i, j-1, \theta_{i, j-1}} \\
= & e_{i j k_{1}}^{0}+\Delta t+\sum_{k_{2}=k_{1}+1}^{\theta_{i j}-1} p_{i j k_{2}}^{0}+\sum_{k_{3}=k_{1}}^{\theta_{i j}-1} u\left(z_{i j k_{3}}^{0}, z_{i, j, k_{3}+1}^{0}\right)-e_{i, j-1, \theta_{i, j-1}}^{0} \\
& -p_{i, j-1, \theta_{i, j-1}}^{\max }+p_{i, j-1, \theta_{i, j-1}}^{0} \\
= & \gamma_{j-1, j}^{i}-p_{i, j-1, \theta_{i, j-1}}^{\max }+p_{i, j-1, \theta_{i, j-1}}^{0}
\end{aligned}
$$

If $p_{i, j-1, \theta_{i, j-1}}^{\max }-p_{i, j-1, \theta_{i, j-1}}^{0}-\gamma_{j-1, j}^{i} \geq 0$, then $\left(\gamma_{j-1, j}^{i}\right)^{\prime} \leq 0$. It can be seen that the planned casting break between $o_{i, j-1, \theta_{i, j-1}}$ and $o_{i, j}, \theta_{i j}$ can be resolved only by adjusting the processing time of $L_{i, j-1}$ at continuous casting stage. So, according to the classification of planned casting break of scheduling plan (section 2.3), the abnormal condition is one-level planned casting break $\zeta_{11}$.

(2) If $p_{i, j-1, \theta_{i, j-1}}^{\max }-p_{i, j-1, \theta_{i, j-1}}^{0}-\gamma_{j-1, j}^{i}<0$, then for the nodes $l \mid l \in V, l \notin V_{i j}$, let $\varepsilon_{l}=0$. If $\tilde{\gamma}_{j-1, j}^{i} \leq 0$, then the abnormal condition of scheduling plan is one-level planned casting break $\zeta_{11}$.

Proof. If $p_{i, j-1, \theta_{i, j-1}}^{\max }-p_{i, j-1, \theta_{i, j-1}}^{0}-\gamma_{j-1, j}^{i}<0$, then $\left(\gamma_{j-1, j}^{i}\right)^{\prime}>0$ according to the formula (28). It can been seen that the planned casting break between $o_{i, j-1, \theta_{i, j-1}}$ and $o_{i, j, \theta_{i j}}$ can not be resolved only by adjusting the processing time of $L_{i, j-1}$ at continuous casting stage. For the nodes $l \mid l \in V, l \notin V_{i j}$, let $\varepsilon_{l}=$ 0 , which means that only nodes belonging to $V_{i j}$ have buffer time. According to the formula (27), $\tilde{\gamma}_{j-1, j}^{i}=\left(s_{i, j, \theta_{i j}}^{0}+\right.$ $\left.\eta_{l_{2}}\right)-\left(e_{i, j-1, \theta_{i, j-1}}^{0}+\lambda_{l_{1}}\right)=\eta_{l_{2}}-\lambda_{l_{1}}$. If $\tilde{\gamma}_{j-1, j}^{i} \leq 0$, then It can be seen that the planned casting break between $o_{i, j-1, \theta_{i, j-1}}$ and $o_{i, j, \theta_{i j}}$ can be resolved only by adjusting the processing time of $L_{i j}$ at each stage. So, according to the classification of planned casting break of scheduling plan (section 2.3), the abnormal condition is one-level planned casting break $\zeta_{11}$.

(3) If $p_{i, j-1, \theta_{i, j-1}}^{\max }-p_{i, j-1, \theta_{i, j-1}}^{0}-\gamma_{j-1, j}^{i}<0$, then for the nodes $l \mid l \in V, l \notin V_{i j}$, let $\varepsilon_{l}=0$. If $\tilde{\gamma}_{j-1, j}^{i}>0$, but $p_{i, j-1, \theta_{i, j-1}}^{\max }-p_{i, j-1, \theta_{i, j-1}}^{0}-$ $\tilde{\gamma}_{j-1, j}^{i} \geq 0$, then the abnormal condition of scheduling plan is two-level planned casting break $\zeta_{12}$.

Proof. If $p_{i, j-1, \theta_{i, j-1}}^{\max }-p_{i, j-1, \theta_{i, j-1}}^{0}-\gamma_{j-1, j}^{i}<0$, then $\left(\gamma_{j-1, j}^{i}\right)^{\prime}>0$, it can been seen that the planned casting break between $o_{i, j-1, \theta_{i, j-1}}$ and $o_{i, j,}, \theta_{i j}$ can not be resolved only by adjusting the processing time of $L_{i, j-1}$ at continuous casting stage. For the nodes $l \mid l \in V, l \notin V_{i j}$, let $\varepsilon_{l}=0$. According to the formula (27), $\tilde{\gamma}_{j-1, j}^{i}=\left(s_{i, j, \theta_{i j}}^{0}+\eta_{l_{2}}\right)-\left(e_{i, j-1, \theta_{i, j-1}}^{0}+\lambda_{l_{1}}\right)=\eta_{l_{2}}-\lambda_{l_{1}}$. If $\tilde{\gamma}_{j-1, j}^{i}>0$, then It can be seen that the planned casting break between $o_{i, j-1, \theta_{i, j-1}}$ and $o_{i, j}, \theta_{i j}$ can not be resolved only by adjusting the processing time of $L_{i j}$ at each stage. If the processing time of operation $o_{i, j-1, \theta_{i, j-1}}$ is adjusted to $p_{i, j-1, \theta_{i, j-1}}$, then the new completion time $e_{i, j-1, \theta_{i, j-1}}$ of operation $o_{i, j-1, \theta_{i, j-1}}$ can be recalculated as follows: $e_{i, j-1, \theta_{i, j-1}}=e_{i, j-1, \theta_{i, j-1}}^{0}+p_{i, j-1, \theta_{i, j-1}}^{\max }-p_{i, j-1, \theta_{i, j-1}}^{0}$. Then according to the formula (27), the new planned casting break time (denoted as $\left.\left(\tilde{\gamma}_{j-1, j}^{i}\right)^{\prime}\right)$ can be recalculated as follows:

$$
\begin{aligned}
\left(\tilde{\gamma}_{j-1, j}^{i}\right)^{\prime} & =\left(s_{i, j, \theta_{i j}}^{0}+\eta_{l_{2}}\right)-\left(e_{i, j-1, \theta_{i, j-1}}^{0}+\lambda_{l_{1}}\right) \\
& =\left(s_{i, j, \theta_{i j}}^{0}+\eta_{l_{2}}\right)-\left(e_{i, j-1, \theta_{i, j-1}}^{0}+p_{i, j-1, \theta_{i, j-1}}^{\max }-p_{i, j-1, \theta_{i, j-1}}^{0}+\lambda_{l_{1}}\right) \\
& =\left(s_{i, j, \theta_{i j}}^{0}+\eta_{l_{2}}\right)-\left(e_{i, j-1, \theta_{i, j-1}}^{0}+\lambda_{l_{1}}\right)-p_{i, j-1, \theta_{i, j-1}}^{\max }+p_{i, j-1, \theta_{i, j-1}}^{0} \\
& =\tilde{\gamma}_{j-1, j}^{i}-p_{i, j-1, \theta_{i, j-1}}^{\max }+p_{i, j-1, \theta_{i, j-1}}^{0}
\end{aligned}
$$

If $p_{i, j-1, \theta_{i j-1}}^{\max }-p_{i, j-1, \theta_{i j-1}}^{0}-\tilde{\gamma}_{j-1, j}^{i} \geq 0$, then $\left(\tilde{\gamma}_{j-1, j}^{i}\right)^{\prime} \leq 0$. It can be seen that the planned casting break between $o_{i, j-1, \theta_{i, j-1}}$ and $o_{i, j}, \theta_{i j}$ can be resolved synchronously by adjusting the processing time of $L_{i, j-1}$ at continuous casting stage and the processing time of $L_{i j}$ at each stage. So, according to the classification of planned casting break of scheduling plan (section 2.3), the abnormal condition is two-level planned casting break $\zeta_{12}$.

(4) If let $\varepsilon_{l}=0$ for the nodes $l \mid l \in V, \quad l \notin V_{i j}, p_{i, j-1, \theta_{i, j-1}}^{\max }-$ $p_{i, j-1, \theta_{i, j-1}}^{0}-\gamma_{j-1, j}^{i}<0, \tilde{\gamma}_{j-1, j}^{i}>0, p_{i, j-1, \theta_{i, j-1}}^{\max }-p_{i, j-1, \theta_{i, j-1}}^{0}-\tilde{\gamma}_{j-1, j}^{i}<0$. If let $\varepsilon_{l} \neq 0$ for the nodes $l \mid l \in V, \tilde{\gamma}_{j-1, j}^{i} \leq 0$, then the abnormal condition of scheduling plan is three-level planned casting break $\zeta_{13}$.

Proof. For the nodes $l \mid l \in V, l \notin V_{i j}$, let $\varepsilon_{l}=0$. If $p_{i, j-1, \theta_{i j-1}}^{\max }$ $p_{i, j-1, \theta_{i, j-1}}^{0}-\gamma_{j-1, j}^{i}<0, \tilde{\gamma}_{j-1, j}^{i}>0, p_{i, j-1, \theta_{i, j-1}}^{\max }-p_{i, j-1, \theta_{i, j-1}}^{0}-\tilde{\gamma}_{j-1, j}^{i}<0$, it means that the planned casting break between $o_{i, j-1, \theta_{i, j-1}}$ and $o_{i, j, \theta_{i j}}$ can not be resolved synchronously by adjusting the processing time of $L_{i, j-1}$ at continuous casting stage and the processing time of $L_{i j}$ at each stage according to the above analysis. If let $\varepsilon_{l} \neq 0$ for the nodes $l \mid l \in V$, then all nodes have duffer time. If $\tilde{\gamma}_{j-1, j}^{i} \leq 0$, then it means that the planned casting break between charge $L_{i, j-1}$ and $L_{i j}$ can be resolved syn- 
chronously by adjusting the processing time of $L_{i j_{1}}\left(j_{1} \leq j\right)$ at each stage. So, according to the classification of planned casting break of scheduling plan (section 2.3), the abnormal condition is three-level planned casting break $\zeta_{13}$.

(5) For the nodes $l \mid l \in V$, if $\tilde{\gamma}_{j-1, j}^{i}>0$, then the abnormal condition of scheduling plan is four-level planned casting break $\zeta_{14}$.

Proof. If let $\varepsilon_{l} \neq 0$ for the nodes $l \mid l \in V$, then all nodes have duffer time. If $\tilde{\gamma}_{j-1, j}^{i}>0$, then it can be known that the planned casting break between charge $L_{i, j-1}$ and $L_{i j}$ can not be resolved synchronously by adjusting the processing time of $L_{i j_{1}}\left(j_{1} \leq j\right)$ at each stage according to the above analysis. In this case, the planned casting break must be resolved synchronously by adjusting the processing machines of $L_{i j_{1}}\left(j_{1} \leq j\right)$ at steelmaking stage and refining stage and by adjusting the processing time of $L_{i j_{1}}\left(j_{1} \leq j\right)$ at each stage. So, according to the classification of planned casting break of scheduling plan (section 2.3), the abnormal condition is four-level planned casting break $\zeta_{14}$.

\subsubsection{The Processing Conflict Decision of Initial Sched-} uling Plan by Operation Time Delay under Buffer Units

When the node $l_{0}\left(l_{0}\right.$ is correspond to the charge $\left.L_{i j}\right)$ delay $\Delta t$, if $\chi_{I(i j k)}^{i j k}>0$, then there is processing conflict time between $o_{i j k}$ and $o_{I(i j k)}$. The $o_{i j k}$ is the corresponding operation for the node $l_{1}$ and the $o_{I(i j k)}$ is the corresponding operation for the node $l_{2}$. We define the $V_{1}$ as the all nodes set for charge $L_{i j}$ and the $V_{2}$ as the all nodes set for charge including $o_{I(i j k)}$. The processing conflict decision is made as follows:

(1) If $\varepsilon_{l_{1}}-\chi_{I(i j k)}^{i j k} \geq 0$, then the abnormal condition of scheduling plan is one-level processing conflict $\zeta_{21}$.

Proof. The operation $o_{I(i j k)}$ is the subsequent operation of the operation $o_{i j k}$ on machine in the initial scheduling plan. When the operation time delay occurs, the processing conflict time $\chi_{I(i j k)}^{i j k}$ can be calculated according to the formula (15). According to the new processing sequence between $o_{i j k}$ and $o_{I(i j k)}$, the processing conflict can be divided into two cases.

- Case 1: $o_{i j k}$ is also processed before $o_{I(i j k)}$ in the new scheduling plan. In this case, the completion time of $o_{i j k}$ is earlier than the completion time of $o_{I(i j k)}$ which means $e_{i j k}<e_{I(i j k)}^{0}$. So, it can be known that $e_{i j k_{1}}+\sum_{k_{2}=k_{1}+1}^{k} p_{i j k_{2}}^{0}+\sum_{k_{3}=k_{1}}^{k-1} u\left(z_{i j k_{3}}^{0}, z_{i, j, k_{3}+1}^{0}\right)<e_{I(i j k)}^{0}$. The processing conflict between $o_{i j k}$ and $o_{I(i j k)}$ may be resolved by adjusting the processing time of $o_{i j k}$. According to the formula (21), $\varepsilon_{l_{1}}=p_{i j k}^{0}-p_{i j k}^{\min }$. If the starting time of operation $o_{i j k}$ is not changed and the processing time of $o_{i j k}$ is adjusted to $p_{i j k}^{\min }$, then according to the formula (15), the new processing conflict time (denoted as $\left.\left(\chi_{(i j k)}^{i j k}\right)^{\prime}\right)$ can be recalculated as follows $\left(k=k_{1}, \ldots, \theta_{i j}\right)$ :

$$
\begin{aligned}
\left(\chi_{I(j i k)}^{i j k}\right)^{\prime} & =\min \left(e_{i j k_{1}}+\sum_{k_{2}=k_{1}+1}^{k-1} p_{i j k_{2}}^{0}+p_{i j k}^{\min }+\sum_{k_{3}=k_{1}}^{k-1} u\left(z_{i j k_{3}}^{0}, z_{i, j, k_{3}+1}^{0}\right), e_{I(i j k)}^{0}\right) \\
& -\max \left(s_{i j k_{1}}+\sum_{k_{2}=k_{1}}^{k-1} p_{i j k_{2}}^{0}+\sum_{k_{3}=k_{1}}^{k-1} u\left(z_{i j k_{3}}^{0}, z_{i, j, k_{3}+1}^{0}\right), s_{I(i j k)}^{0}\right) \\
= & \min \left(e_{i j k_{1}}+\sum_{k_{2}=k_{1}+1}^{k-1} p_{i j k_{2}}^{0}+p_{i j k}^{0}-\varepsilon_{l_{1}}+\sum_{k_{3}=k_{1}}^{k-1} u\left(z_{i j k_{3}}^{0}, z_{i, j, k_{3}+1}^{0}\right), e_{I(i j k)}^{0}\right) \\
& -\max \left(s_{i j k_{1}}+\sum_{k_{2}=k_{1}}^{k-1} p_{i j k_{2}}^{0}+\sum_{k_{3}=k_{1}}^{k-1} u\left(z_{i j k_{3}}^{0}, z_{i, j, k_{3}+1}^{0}\right), s_{I(i j k)}^{0}\right)
\end{aligned}
$$

Because $e_{i j k_{1}}+\sum_{k_{2}=k_{1}+1}^{k} p_{i j k_{2}}^{0}+\sum_{k_{3}=k_{1}}^{k-1} u\left(z_{i j k_{3}}^{0}, z_{i, j, k_{3}+1}^{0}\right)<e_{I(j i k)}^{0}, e_{i j k_{1}}+\sum_{k_{2}=k_{1}+1}^{k}$ $p_{i j k_{2}}^{0}+\sum_{k_{3}=k_{1}}^{k-1} u\left(z_{i j k_{3}}^{0}, z_{i, j, k_{3}+1}^{0}\right)-\varepsilon_{l_{l}}<e_{I(i j k)}^{0}$. So the minimum value of first term in the formula (30) is $e_{i j k_{1}}+\sum_{k_{2}=k_{1}+1}^{k-1} p_{i j k_{2}}^{0}+p_{i j k}^{0}-\varepsilon_{l_{1}}+$ $\sum_{k_{3}=k_{1}}^{k-1} u\left(z_{i j k_{3}}^{0}, z_{i, j, k_{3}+1}^{0}\right)$. Then the formula (30) can be rewritten as follows:

$$
\begin{aligned}
\left(\chi_{I(i j k)}^{i j k}\right)^{\prime} & =\left(\min \left(e_{i j k_{1}}+\sum_{k_{2}=k_{1}+1}^{k} p_{i j k_{2}}^{0}+\sum_{k_{3}=k_{1}}^{k-1} u\left(z_{i j k_{3}}^{0}, z_{i, j, k_{3}+1}^{0}\right), e_{I(i j k)}^{0}\right)-\varepsilon_{l_{1}}\right) \\
& -\max \left(s_{i j k_{1}}+\sum_{k_{2}=k_{1}}^{k-1} p_{i j k_{2}}^{0}+\sum_{k_{3}=k_{1}}^{k-1} u\left(z_{i j k_{3}}^{0}, z_{i, j, k_{3}+1}^{0}\right), s_{I(i j k)}^{0}\right) \\
= & \min \left(e_{i j k_{1}}+\sum_{k_{2}=k_{1}+1}^{k} p_{i j k_{2}}^{0}+\sum_{k_{3}=k_{1}}^{k-1} u\left(z_{i j k_{3}}^{0}, z_{i, j, k_{3}+1}^{0}\right), e_{I(i j k)}^{0}\right) \\
& -\max \left(s_{i j k_{1}}+\sum_{k_{2}=k_{1}}^{k-1} p_{i j k_{2}}^{0}+\sum_{k_{3}=k_{1}}^{k-1} u\left(z_{i j k_{3}}^{0}, z_{i, j, k_{3}+1}^{0}\right), s_{I(i j k)}^{0}\right)-\varepsilon_{l_{1}} \\
= & \chi_{I(i j k)}^{i j k}-\varepsilon_{l_{1}}
\end{aligned}
$$

If $\varepsilon_{l_{1}}-\chi_{I(i j k)}^{i j k} \geq 0$, then $\left(\chi_{I_{(i j k)}^{i j k}}\right)^{\prime} \leq 0$. It can resolve the processing conflict between $o_{i j k}$ and $o_{I(i j k)}$ only by adjusting the processing time of $o_{i j k}$. So, according to the classification of processing conflict of scheduling plan (section 2.3), the abnormal condition is one-level processing conflict $\zeta_{21}$.

- Case 2: $o_{i j k}$ is processed after $o_{I(i j k)}$ in the new scheduling plan. In this case, the starting time of $o_{i j k}$ is later than the starting time of $o_{I(i j k)}$ which means $s_{i j k}>s_{I(i j k)}^{0}$. So, it can be known that $s_{i j k_{1}}+\sum_{k_{2}=k_{1}}^{k-1} p_{i j k_{2}}^{0}+\sum_{k_{3}=k_{1}}^{k-1} u\left(z_{i j k_{3}}^{0}, z_{i, j, k_{3}+1}^{0}\right)>s_{I(j i k)}^{0}$. According to the formula (21), $\varepsilon_{l_{1}}=p_{i j k}^{0}-p_{i j k}^{\min }$. If the completion time of operation $o_{i j k}$ is not changed and the processing time of $o_{i j k}$ is adjusted to $p_{i j k}^{\min }$, then according to the formula (15), the new processing conflict time (denoted as $\left.\left(\chi_{I(i j k)}^{i j k}\right)^{\prime}\right)$ can be recalculated as follows $\left(k=k_{1}, \ldots, \theta_{i j}\right)$ :

$$
\begin{aligned}
\left(\chi_{I(i j k)}^{i j k}\right)^{\prime}= & \min \left(e_{i j k_{1}}+\sum_{k_{2}=k_{1}+1}^{k} p_{i j k_{2}}^{0}+\sum_{k_{3}=k_{1}}^{k-1} u\left(z_{i j k_{3}}^{0}, z_{i, j, k_{3}+1}^{0}\right), e_{I(i j k)}^{0}\right) \\
& -\max \left(s_{i j k_{1}}+\sum_{k_{2}=k_{1}}^{k-1} p_{i j k_{2}}^{0}+\sum_{k_{3}=k_{1}}^{k-1} u\left(z_{i j k_{3}}^{0}, z_{i, j, k_{3}+1}^{0}\right)+\varepsilon_{l_{1}}, s_{I(i j k)}^{0}\right)
\end{aligned}
$$

Because $s_{i j k_{1}}+\sum_{k_{2}=k_{1}}^{k-1} p_{i j k_{2}}^{0}+\sum_{k_{3}=k_{1}}^{k-1} u\left(z_{i j k_{3}}^{0}, z_{i, j, k_{3}+1}^{0}\right)>s_{I(i j k)}^{0}, s_{i j k_{1}}+\sum_{k_{2}=k_{1}}^{k-1} p_{i j k_{2}}^{0}+$ $\sum_{k_{3}=k_{1}}^{k-1} u\left(z_{i j k_{3}}^{0}, z_{i, j, k_{3}+1}^{0}\right)+\varepsilon_{l_{1}}>s_{I(i j k)}^{0}$. So the maximum value of second term in the formula (32) is $s_{i j k_{1}}+\sum_{k_{2}=k_{1}}^{k-1} p_{i j k_{2}}^{0}+\sum_{k_{3}=k_{1}}^{k-1} u\left(z_{i j k_{3}}^{0}, z_{i, j, k_{3}+1}^{0}\right)+$ $\varepsilon_{l_{1}}$. Then the formula (32) can be rewritten as follows:

$$
\begin{aligned}
\left(\chi_{I(j i k)}^{i j k}\right)^{\prime} & =\min \left(e_{i j k_{1}}+\sum_{k_{2}=k_{1}+1}^{k} p_{i j k_{2}}^{0}+\sum_{k_{3}=k_{1}}^{k-1} u\left(z_{i j k_{3}}^{0}, z_{i, j, k_{3}+1}^{0}\right), e_{I(i j k)}^{0}\right) \\
& -\left(\max \left(s_{i j k_{1}}+\sum_{k_{2}=k_{1}}^{k-1} p_{i j k_{2}}^{0}+\sum_{k_{3}=k_{1}}^{k-1} u\left(z_{i j k_{3}}^{0}, z_{i, j, k_{3}+1}^{0}\right), s_{I(i j k)}^{0}\right)+\varepsilon_{l_{1}}\right) \\
& =\min \left(e_{i j k_{1}}+\sum_{k_{2}=k_{1}+1}^{k} p_{i j k_{2}}^{0}+\sum_{k_{3}=k_{1}}^{k-1} u\left(z_{i j k_{3}}^{0}, z_{i, j, k_{3}+1}^{0}\right), e_{I(i j k)}^{0}\right)
\end{aligned}
$$




$$
\begin{aligned}
& -\max \left(s_{i j k_{1}}+\sum_{k_{2}=k_{1}}^{k-1} p_{i j k_{2}}^{0}+\sum_{k_{3}=k_{1}}^{k-1} u\left(z_{i j k_{3}}^{0}, z_{i, j, k_{3}+1}^{0}\right), s_{I(i j k)}^{0}\right)-\varepsilon_{l_{1}} \\
& =\chi_{I(j k k)}^{i j k}-\varepsilon_{l_{1}}
\end{aligned}
$$

If $\varepsilon_{l_{1}}-\chi_{I(i j k)}^{i j k} \geq 0$, then $\left(\chi_{I(i j k)}^{i j k}\right)^{\prime} \leq 0$. It can resolve the processing conflict between $o_{i j k}$ and $o_{I(i j k)}$ only by adjusting the processing time of $o_{i j k}$. So, according to the classification of processing conflict of scheduling plan (section 2.3), the abnormal condition is one-level processing conflict $\zeta_{21}$.

To sum up, if $\varepsilon_{l_{1}}-\chi_{I_{(i j k)}^{i j k}} \geq 0$, then the abnormal condition of scheduling plan is one-level processing conflict $\zeta_{21}$.

(2) If $\varepsilon_{l_{2}}-\chi_{I(i j k)}^{i j k} \geq 0$, then the abnormal condition of scheduling plan is one-level processing conflict $\zeta_{21}$.

Proof. The operation $o_{I(i j k)}$ is the subsequent operation of the operation $o_{i j k}$ on machine in the initial scheduling plan. When the operation time delay occurs, the processing conflict time $\chi_{I(i j k)}^{i j k}$ can be calculated according to the formula (15). According to the new processing sequence between $o_{i j k}$ and $o_{I(i j k)}$, the processing conflict can be divided into two cases.

- Case 1: $o_{i j k}$ is also processed before $o_{I(i j k)}$ in the new scheduling plan. In this case, the starting time of $o_{I(i j k)}$ is later than the starting time of $o_{i j k}$ which means $s_{I(i j k)}^{0}>s_{i j k}$. So, it can be known that $s_{I(i j k)}^{0}>s_{i j k_{1}}+\sum_{k_{2}=k_{1}}^{k-1} p_{i j k_{2}}^{0}+\sum_{k_{3}=k_{1}}^{k-1} u\left(z_{i j k_{3}}^{0}, z_{i, j, k_{3}+1}^{0}\right)$. The processing conflict between $o_{i j k}$ and $o_{I(i j k)}$ may be resolved by adjusting the processing time of $o_{I(i j k)}$. According to the formula (21), $\varepsilon_{l_{2}}=p_{I(i j k)}^{0}-p_{I(i j k)}^{\min }$. If the completion time of operation $o_{I(i j k)}$ is not changed and the processing time of $o_{I(j i k)}$ is adjusted to $p_{I(i j k)}^{\min }$, then according to the formula (15), the new processing conflict time (denoted as $\left(\chi_{I_{(i j k)}^{i j k}}\right)$ ') can be recalculated as follows $\left(k=k_{1}, \ldots, \theta_{i j}\right)$ :

$$
\begin{aligned}
& \left(\chi_{I(i j k)}^{i j k}\right)^{\prime}=\min \left(e_{i j k_{1}}+\sum_{k_{2}=k_{1}+1}^{k} p_{i j k_{2}}^{0}+\sum_{k_{3}=k_{1}}^{k-1} u\left(z_{i j k_{3}}^{0}, z_{i, j, k_{3}+1}^{0}\right), e_{I(i j k)}^{0}\right) \\
& -\max \left(s_{i j k_{1}}+\sum_{k_{2}=k_{1}}^{k-1} p_{i j k_{2}}^{0}+\sum_{k_{3}=k_{1}}^{k-1} u\left(z_{i j k_{3}}^{0}, z_{i, j, k_{3}+1}^{0}\right), s_{I(i j k)}^{0}+\varepsilon_{l_{2}}\right)
\end{aligned}
$$

Because $s_{I(i j k)}^{0}>s_{i j k_{1}}+\sum_{k_{2}=k_{1}}^{k-1} p_{i j k_{2}}^{0}+\sum_{k_{3}=k_{1}}^{k-1} u\left(z_{i j k_{3}}^{0}, z_{i, j, k_{3}+1}^{0}\right), s_{I(i j k)}^{0}+\varepsilon_{l_{2}}>$ $s_{i j k_{1}}+\sum_{k_{2}=k_{1}}^{k-1} p_{i j k_{2}}^{0}+\sum_{k_{3}=k_{1}}^{k-1} u\left(z_{i j k_{3}}^{0}, z_{i, j, k_{3}+1}^{0}\right)$. So the maximum value of second term in the formula (34) is $s_{I(i j))}^{0}+\varepsilon_{l_{2}}$. Then the formula (34) can be rewritten as follows:

$$
\begin{aligned}
\left(\chi_{I(i j k)}^{i j k}\right)^{\prime}= & \min \left(e_{i j k_{1}}+\sum_{k_{2}=k_{1}+1}^{k} p_{i j k_{2}}^{0}+\sum_{k_{3}=k_{1}}^{k-1} u\left(z_{i j k_{3}}^{0}, z_{i, j, k_{3}+1}^{0}\right), e_{I(i j k)}^{0}\right) \\
& -\left(\max \left(s_{i j k_{1}}+\sum_{k_{2}=k_{1}}^{k-1} p_{i j k_{2}}^{0}+\sum_{k_{3}=k_{1}}^{k-1} u\left(z_{i j k_{3}}^{0}, z_{i, j, k_{3}+1}^{0}\right), s_{I(i j k)}^{0}\right)+\varepsilon_{l_{2}}\right) \\
& =\min \left(e_{i j k_{1}}+\sum_{k_{2}=k_{1}+1}^{k} p_{i j k_{2}}^{0}+\sum_{k_{3}=k_{1}}^{k-1} u\left(z_{i j k_{3}}^{0}, z_{i, j, k_{3}+1}^{0}\right), e_{I(i j k)}^{0}\right) \\
& -\max \left(s_{i j k_{1}}+\sum_{k_{2}=k_{1}}^{k-1} p_{i j k_{2}}^{0}+\sum_{k_{3}=k_{1}}^{k-1} u\left(z_{i j k_{3}}^{0}, z_{i, j, k_{3}+1}^{0}\right), s_{I(i j k)}^{0}\right)-\varepsilon_{l_{2}} \\
= & \chi_{I(i j k)}^{i j k}-\varepsilon_{l_{2}}
\end{aligned}
$$

If $\varepsilon_{l_{2}}-\chi_{I(j k k)}^{i j k} \geq 0$, then $\left(\chi_{I(j i k)}^{i j k}\right)^{\prime} \leq 0$. It can resolve the process- ing conflict between $o_{i j k}$ and $o_{I(i j k)}$ only by adjusting the processing time of $o_{I(i j k)}$. So, according to the classification of processing conflict of scheduling plan (section 2.3), the abnormal condition is one-level processing conflict $\zeta_{21}$.

- Case 2: $o_{i j k}$ is processed after $o_{I(i j k)}$ in the new scheduling plan. In this case, the completion time of $o_{I(i j k)}$ is earlier than the completion time of $o_{i j k}$ which means $e_{I(i j k)}^{0}<e_{i j k}$. So, it can be known that $e_{I(i j k)}^{0}<e_{i j k_{1}}+\sum_{k_{2}=k_{1}+1}^{k} p_{i j k_{2}}^{0}+\sum_{k_{3}=k_{1}}^{k-1} u\left(z_{i j k_{3}}^{0}, z_{i, j, k_{3}+1}^{0}\right)$. The processing conflict between $o_{i j k}$ and $o_{I(i j k)}$ may be resolved by adjusting the processing time of $o_{I(i j k)}$. According to the formula (21), $\varepsilon_{l_{2}}=p_{I(i j k)}^{0}-p_{I(i j k)}^{\min }$. If the starting time of operation $o_{I(i j k)}$ is not changed and the processing time of $o_{I(i j k)}$ is adjusted to $p_{I(i j k)}^{\min }$, then according to the formula (15), the new processing conflict time (denoted as $\left.\left(\chi_{I(i j k)}^{i j k}\right)^{\prime}\right)$ can be recalculated as follows $\left(k=k_{1}, \ldots, \theta_{i j}\right)$ :

$$
\begin{aligned}
& \left(\chi_{I(i j k)}^{i j k}\right)^{\prime}=\min \left(e_{i j k_{1}}+\sum_{k_{2}=k_{1}+1}^{k} p_{i j k_{2}}^{0}+\sum_{k_{3}=k_{1}}^{k-1} u\left(z_{i j k_{3}}^{0}, z_{i, j, k_{3}+1}^{0}\right), e_{I(i j k)}^{0}-\varepsilon_{l_{2}}\right) \\
& -\max \left(s_{i j k_{1}}+\sum_{k_{2}=k_{1}}^{k-1} p_{i j k_{2}}^{0}+\sum_{k_{3}=k_{1}}^{k-1} u\left(z_{i j k_{3}}^{0}, z_{i, j, k_{3}+1}^{0}\right), s_{I(i j k)}^{0}\right)
\end{aligned}
$$

Because $e_{I(i j k)}^{0}<e_{i j k_{1}}+\sum_{k_{2}=k_{1}+1}^{k} p_{i j k_{2}}^{0}+\sum_{k_{3}=k_{1}}^{k-1} u\left(z_{i j k_{3}}^{0}, z_{i, j, k_{3}+1}^{0}\right), \quad e_{I(i j k)}^{0}-\varepsilon_{l_{2}}<$ $e_{i j k_{1}}+\sum_{k_{2}=k_{1}+1}^{k} p_{i j k_{2}}^{0}+\sum_{k_{3}=k_{1}}^{k-1} u\left(z_{i j k_{3}}^{0}, z_{i, j, k_{3}+1}^{0}\right)$. So the minimum value of first term in the formula (36) is $e_{I(i j k)}^{0}-\varepsilon_{l_{2}}$. Then the formula (36) can be rewritten as follows:

$$
\begin{aligned}
\left(\chi_{I(j i k)}^{i j k}\right) & =\left(\min \left(e_{i j k_{1}}+\sum_{k_{2}=k_{1}+1}^{k} p_{i j k_{2}}^{0}+\sum_{k_{3}=k_{1}}^{k-1} u\left(z_{i j k_{3}}^{0}, z_{i, j, k_{3}+1}^{0}\right), e_{I(j i k)}^{0}\right)-\varepsilon_{l_{2}}\right) \\
& -\max \left(s_{i j k_{1}}+\sum_{k_{2}=k_{1}}^{k-1} p_{i j k_{2}}^{0}+\sum_{k_{3}=k_{1}}^{k-1} u\left(z_{i j k_{3}}^{0}, z_{i, j, k_{3}+1}^{0}\right), s_{I(j i k)}^{0}\right) \\
& =\min \left(e_{i j k_{1}}+\sum_{k_{2}=k_{1}+1}^{k} p_{i j k_{2}}^{0}+\sum_{k_{3}=k_{1}}^{k-1} u\left(z_{i j k_{3}}^{0}, z_{i, j, k_{3}+1}^{0}\right), e_{I(j i k)}^{0}\right) \\
& -\max \left(s_{i j k_{1}}+\sum_{k_{2}=k_{1}}^{k-1} p_{i j k_{2}}^{0}+\sum_{k_{3}=k_{1}}^{k-1} u\left(z_{i j k_{3}}^{0}, z_{i, j, k_{3}+1}^{0}\right), s_{I(i j k)}^{0}\right)-\varepsilon_{l_{2}} \\
& =\chi_{I(j i j k)}^{i j k}-\varepsilon_{l_{2}}
\end{aligned}
$$

If $\varepsilon_{l_{2}}-\chi_{I(j i k)}^{i j k} \geq 0$, then $\left(\chi_{I(i j k)}^{i j k}\right)^{\prime} \leq 0$. It can resolve the processing conflict between $o_{i j k}$ and $o_{I(i j k)}$ only by adjusting the processing time of $o_{I(i j k)}$. So, according to the classification of processing conflict of scheduling plan (section 2.3), the abnormal condition is one-level processing conflict $\zeta_{21}$.

To sum up, if $\varepsilon_{l_{2}}-\chi_{I(i j k)}^{i j k} \geq 0$, then the abnormal condition of scheduling plan is one-level processing conflict $\zeta_{21}$.

(3) If $\varepsilon_{l_{1}}-\chi_{I(i j k)}^{i j k}<0, \varepsilon_{l_{2}}-\chi_{I(i j k)}^{i j k}<0, \varepsilon_{l_{1}}+\varepsilon_{l_{2}}-\chi_{I(i j k)}^{i j k} \geq 0$, then the abnormal condition of scheduling plan is two-level processing conflict $\zeta_{22}$.

Proof. The operation $o_{I(i j k)}$ is the subsequent operation of the operation $o_{i j k}$ on machine in the initial scheduling plan. When the operation time delay occurs, the processing conflict time $\chi_{I(j i k)}^{i j k}$ can be calculated according to the formula (15). According to above analysis, if $\varepsilon_{l_{1}}-\chi_{I(i j k)}^{i j k}<0$, it can not resolve the processing conflict between $o_{i j k}$ and $o_{I(i j k)}$ only by adjusting the processing time of $o_{i j k}$. If $\varepsilon_{l_{2}}-\chi_{I(i j k)}^{i j k}<$ 0 , it can not resolve the processing conflict between $o_{i j k}$ and $o_{I(i j k)}$ only by adjusting the processing time of $o_{I(i j k)}$. According to the new processing sequence between $o_{i j k}$ and $o_{I(i j k)}$, the processing conflict can be divided into two cases.

- Case 1: $o_{i j k}$ is also processed before $o_{I(i j k)}$ in the new sched- 
uling plan. In this case, the completion time of $o_{i j k}$ is earlier than the completion time of $o_{I(i j k)}$ which means $e_{i j k}<e_{I(i j k)}^{0}$. So, it can be known that $e_{i j k_{1}}+\sum_{k_{2}=k_{1}+1}^{k} p_{i j k_{2}}^{0}+\sum_{k_{3}=k_{1}}^{k-1} u\left(z_{i j k_{3}}^{0}, z_{i, j, k_{3}+1}^{0}\right)<e_{I(i j k)}^{0}$. The starting time of $o_{I(i j k)}$ is later than the starting time of $o_{i j k}$ which means $s_{I(i j k)}^{0}>s_{i j k}$. So, it can be known that $s_{I(i j k)}^{0}>$ $s_{i j k_{1}}+\sum_{k_{2}=k_{1}}^{k-1} p_{i j k_{2}}^{0}+\sum_{k_{3}=k_{1}}^{k-1} u\left(z_{i j k_{3}}^{0}, z_{i, j, k_{3}+1}^{0}\right)$. The processing conflict may be resolved synchronously by adjusting the processing time of $o_{i j k}$ and $o_{I(i j k)}$. According to the formula (21), $\varepsilon_{l_{1}}=p_{i j k}^{0}-p_{i j k}^{\min }$ and $\varepsilon_{l_{2}}=p_{I(i j k)}^{0}-p_{I(j i k)}^{\min }$. If the starting time of operation $o_{i j k}$ is not changed and the processing time of $o_{i j k}$ is adjusted to, and if the completion time of operation $o_{I(i j k)}$ is not changed and the processing time of $o_{I(i j k)}$ is adjusted to $p_{I(i j k)}^{\mathrm{min}}$, then according to the formula (15), the new processing conflict time (denoted as $\left.\left(\chi_{I(i j k)}^{i j k}\right)^{\prime}\right)$ can be recalculated as follows $\left(k=k_{1}, \ldots, \theta_{i j}\right)$ :

$$
\begin{aligned}
& \left(\chi_{I(i j k)}^{i j k}\right)^{\prime} \\
& =\min \left(e_{i j k_{1}}+\sum_{k_{2}=k_{1}+1}^{k-1} p_{i j k_{2}}^{0}+p_{i j k}^{0}-\varepsilon_{l_{1}}+\sum_{k_{3}=k_{1}}^{k-1} u\left(z_{i j k_{3}}^{0}, z_{i, j, k_{3}+1}^{0}\right), e_{I(i j k)}^{0}\right) \\
& -\max \left(s_{i j k_{1}}+\sum_{k_{2}=k_{1}}^{k-1} p_{i j k_{2}}^{0}+\sum_{k_{3}=k_{1}}^{k-1} u\left(z_{i j k_{3}}^{0}, z_{i, j, k_{3}+1}^{0}\right), s_{I(j i k)}^{0}+\varepsilon_{l_{2}}\right)
\end{aligned}
$$

Because $e_{i j k_{1}}+\sum_{k_{2}=k_{1}+1}^{k} p_{i j k_{2}}^{0}+\sum_{k_{3}=k_{1}}^{k-1} u\left(z_{i j k_{3}}^{0}, z_{i, j, k_{3}+1}^{0}\right)<e_{I(i j k)}^{0}$ and $s_{I(j i k)}^{0}>$ $s_{i j k_{1}}+\sum_{k_{2}=k_{1}}^{k-1} p_{i j k_{2}}^{0}+\sum_{k_{3}=k_{1}}^{k-1} u\left(z_{i j k_{3}}^{0} z_{i, j, k_{3}+1}^{0}\right), e_{i j k_{1}}+\sum_{k_{2}=k_{1}+1}^{k} p_{i j k_{2}}^{0}+\sum_{k_{3}=k_{1}}^{k-1} u\left(z_{i j k_{3}}^{0}, z_{i, j, k_{3}+1}^{0}\right)-$ $\varepsilon_{l_{1}}<e_{I(j i k)}^{0}$ and $s_{I(j i k)}^{0}+\varepsilon_{l_{2}}>s_{i j k_{1}}+\sum_{k_{2}=k_{1}}^{k-1} p_{i j k_{2}}^{0}+\sum_{k_{3}=k_{1}}^{k-1} u\left(z_{i j k_{3}}^{0}, z_{i, j, k_{3}+1}^{0}\right)$. Then the formula (38) can be rewritten as follows:

$$
\begin{aligned}
\left(\chi_{I(j i j)}^{i j k}\right)^{\prime} & =\left(\min \left(e_{i j k_{1}}+\sum_{k_{2}=k_{1}+1}^{k-1} p_{i j k_{2}}^{0}+p_{i j k}^{0}+\sum_{k_{3}=k_{1}}^{k-1} u\left(z_{i j k_{3}}^{0}, z_{i, j, k_{3}+1}^{0}\right), e_{I(i j k)}^{0}\right)-\varepsilon_{l_{1}}\right) \\
& -\left(\max \left(s_{i j k_{1}}+\sum_{k_{2}=k_{1}}^{k-1} p_{i j k_{2}}^{0}+\sum_{k_{3}=k_{1}}^{k-1} u\left(z_{i j k_{3}}^{0}, z_{i, j, k_{3}+1}^{0}\right), s_{I(j i k)}^{0}\right)+\varepsilon_{l_{2}}\right) \\
= & \min \left(e_{i j k_{1}}+\sum_{k_{2}=k_{1}+1}^{k-1} p_{i j k_{2}}^{0}+p_{i j k}^{0}+\sum_{k_{3}=k_{1}}^{k-1} u\left(z_{i j k_{3}}^{0}, z_{i, j, k_{3}+1}^{0}\right), e_{I(i j k)}^{0}\right) \\
& -\max \left(s_{i j k_{1}}+\sum_{k_{2}=k_{1}}^{k-1} p_{i j k_{2}}^{0}+\sum_{k_{3}=k_{1}}^{k-1} u\left(z_{i j k_{3}}^{0}, z_{i, j, k_{3}+1}^{0}\right), s_{I(i j k)}^{0}\right)-\varepsilon_{l_{1}}-\varepsilon_{l_{2}} \\
= & \chi_{I(i j k)}^{i j k}-\varepsilon_{l_{1}}-\varepsilon_{l_{2}}
\end{aligned}
$$

If $\varepsilon_{l_{1}}+\varepsilon_{l_{2}}-\chi_{I(i j k)}^{i j k} \geq 0$, then $\left(\chi_{I(i j k)}^{i j k}\right)^{\prime} \leq 0$. It can resolve the processing conflict between $o_{i j k}$ and $o_{I(i j k)}$ synchronously by adjusting the processing time of $o_{i j k}$ and $o_{I(i j k)}$. So, according to the classification of processing conflict of scheduling plan (section 2.3), the abnormal condition is one-level processing conflict $\zeta_{22}$.

- Case 2: $o_{i j k}$ is processed after $o_{I(i j k)}$ in the new scheduling plan. It can be known that $s_{i j k_{1}}+\sum_{k_{2}=k_{1}}^{k-1} p_{i j k_{2}}^{0}+\sum_{k_{3}=k_{1}}^{k-1} u\left(z_{i j k_{3}}^{0}, z_{i, j, k_{3}+1}^{0}\right)>$ $s_{I(i j k)}^{0}$ and $e_{I(i j k)}^{0}<e_{i j k_{1}}+\sum_{k_{2}=k_{1}+1}^{k} p_{i j k_{2}}^{0}+\sum_{k_{3}=k_{1}}^{k-1} u\left(z_{i j k_{3}}^{0}, z_{i, j, k_{3}+1}^{0}\right)$. If the completion time of operation $o_{i j k}$ is not changed and the processing time of $o_{i j k}$ is adjusted to $p_{i j k}^{\min }$, and if the starting time of operation $o_{I(i j k)}$ is not changed and the processing time of
$o_{I(i j k)}$ is adjusted to $p_{I(i j k)}^{\min }$, then according to the formula (15), the new processing conflict time (denoted as $\left(\chi_{I(i j k)}^{i j k}\right)$ ) can be recalculated as follows $\left(k=k_{1}, \ldots, \theta_{i j}\right)$ :

$$
\begin{aligned}
\left(\chi_{I(i j k)}^{i j k}\right)^{\prime} & =\min \left(e_{i j k_{1}}+\sum_{k_{2}=k_{1}+1}^{k} p_{i j k_{2}}^{0}+\sum_{k_{3}=k_{1}}^{k-1} u\left(z_{i j k_{3}}^{0}, z_{i, j, k_{3}+1}^{0}\right), e_{I(i j k)}^{0}-\varepsilon_{l_{2}}\right) \\
& -\max \left(s_{i j k_{1}}+\sum_{k_{2}=k_{1}}^{k-1} p_{i j k_{2}}^{0}+\sum_{k_{3}=k_{1}}^{k-1} u\left(z_{i j k_{3}}^{0}, z_{i, j, k_{3}+1}^{0}\right)+\varepsilon_{l_{1}}, s_{I(i j k)}^{0}\right)
\end{aligned}
$$

Because $e_{I(i j k)}^{0}<e_{i j k_{1}}+\sum_{k_{2}=k_{1}+1}^{k} p_{i j k_{2}}^{0}+\sum_{k_{3}=k_{1}}^{k-1} u\left(z_{i j k_{3}}^{0}, z_{i, j, k_{3}+1}^{0}\right)$ and $s_{i j k_{1}}+$ $\sum_{k_{2}=k_{1}}^{k-1} p_{i j k_{2}}^{0}+\sum_{k_{3}=k_{1}}^{k-1} u\left(z_{i j k_{3}}^{0}, z_{i, j, k_{3}+1}^{0}\right)>s_{I(i j k)}^{0}, \quad e_{I(i j k)}^{0}-\varepsilon_{l_{2}}<e_{i j k_{1}}+\sum_{k_{2}=k_{1}+1}^{k} p_{i j k_{2}}^{0}+$ $\sum_{k_{3}=k_{1}}^{k-1} u\left(z_{i j k_{3}}^{0}, z_{i, j, k_{3}+1}^{0}\right)$ and $s_{i j k_{1}}+\sum_{k_{2}=k_{1}}^{k-1} p_{i j k_{2}}^{0}+\sum_{k_{3}=k_{1}}^{k-1} u\left(z_{i j k_{3}}^{0}, z_{i, j, k_{3}+1}^{0}\right)+\varepsilon_{l_{1}}>s_{I(i j k)}^{0}$.

Then the formula (40) can be rewritten as follows:

$$
\begin{aligned}
\left(\chi_{I(i j k)}^{i j k}\right)^{\prime} & =\left(\min \left(e_{i j k_{1}}+\sum_{k_{2}=k_{1}+1}^{k} p_{i j k_{2}}^{0}+\sum_{k_{3}=k_{1}}^{k-1} u\left(z_{i j k_{3}}^{0}, z_{i, j, k_{3}+1}^{0}\right), e_{I(i j k)}^{0}\right)-\varepsilon_{l_{2}}\right) \\
& -\left(\max \left(s_{i j k_{1}}+\sum_{k_{2}=k_{1}}^{k-1} p_{i j k_{2}}^{0}+\sum_{k_{3}=k_{1}}^{k-1} u\left(z_{i j k_{3}}^{0}, z_{i, j, k_{3}+1}^{0}\right), s_{I(i j k)}^{0}\right)+\varepsilon_{l_{1}}\right) \\
& =\min \left(e_{i j k_{1}}+\sum_{k_{2}=k_{1}+1}^{k} p_{i j k_{2}}^{0}+\sum_{k_{3}=k_{1}}^{k-1} u\left(z_{i j k_{3}}^{0}, z_{i, j, k_{3}+1}^{0}\right), e_{I(i j k)}^{0}\right) \\
& -\max \left(s_{i j k_{1}}+\sum_{k_{2}=k_{1}}^{k-1} p_{i j k_{2}}^{0}+\sum_{k_{3}=k_{1}}^{k-1} u\left(z_{i j k_{3}}^{0}, z_{i, j, k_{3}+1}^{0}\right), s_{I(i j k)}^{0}\right)-\varepsilon_{l_{1}}-\varepsilon_{l_{2}} \\
& =\chi_{I(i j k)}^{i i j k}-\varepsilon_{l_{1}}-\varepsilon_{l_{2}}
\end{aligned}
$$

If $\varepsilon_{l_{1}}+\varepsilon_{l_{2}}-\chi_{I(i j k)}^{i j k} \geq 0$, then $\left(\chi_{I(i j k)}^{i j k}\right)^{\prime} \leq 0$. It can resolve the processing conflict between $o_{i j k}$ and $o_{I(j i k)}$ synchronously by adjusting the processing time of $o_{i j k}$ and $o_{I(i j k)}$. So, according to the classification of processing conflict of scheduling plan (section 2.3), the abnormal condition is one-level processing conflict $\zeta_{22}$.

To sum up, If $\varepsilon_{l_{1}}-\chi_{I(i j k)}^{i j k}<0, \varepsilon_{l_{2}}-\chi_{I(i j k)}^{i j k}<0, \varepsilon_{l_{1}}+\varepsilon_{l_{2}}-\chi_{I(i j k)}^{i j k} \geq$ 0 , then the abnormal condition of scheduling plan is twolevel processing conflict $\zeta_{22}$.

(4) If $\varepsilon_{l_{1}}+\varepsilon_{l_{2}}-\chi_{I(i j k)}^{i j k}<0$, then let $\varepsilon_{l}=0$ for the nodes $l \mid l \in$ $V, l \notin V_{1}, l \notin V_{2}$. If $\sum_{l_{1}^{*} \in V_{1}^{*}} \varepsilon_{l_{1}^{*}}+\sum_{l_{2}^{*} \in V_{2}^{*}} \varepsilon_{l_{2}^{*}}-\chi_{I(j i k)}^{i j k} \geq 0$, then the abnormal condition of scheduling plan is three-level processing conflict $\zeta_{23}$.

Proof. According to above analysis, if $\varepsilon_{l_{1}}+\varepsilon_{l_{2}}-\chi_{I(i j k)}^{i j k}<0$, It can not resolve the processing conflict between $o_{i j k}$ and $o_{I(i j k)}$ synchronously by adjusting the processing time of $o_{i j k}$ and $o_{I(i j k)}$. We define $o_{I(i j k)}$ is the $k^{1}$ th operation of the charge $L_{i^{1} j^{1}}$, so the $o_{I(i j k)}$ can also be denoted as $o_{i^{1} j^{1} k^{1}}$. We define the $V_{1}^{*}$ as the all nodes set for $o_{i j k_{1}^{*}} \mid k_{1}^{*} \leq k$ of $L_{i j}$ and the $V_{2}^{*}$ as the all nodes set for $O_{i^{1} j^{1} k_{2}^{*}} \mid k_{2}^{*} \leq k^{1}$ of $L_{i^{1} j^{1}}$. The processing conflict may be resolved synchronously by adjusting the processing time of operations in $V_{1}^{*}$ and $V_{2}^{*}$. According to the new processing sequence between $o_{i j k}$ and $o_{I(i j k)}$, the processing conflict can be divided into two cases.

- Case 1: $o_{i j k}$ is also processed before $o_{I(i j k)}$ in the new scheduling plan. It can be known that $e_{i j k_{1}}+\sum_{k_{2}=k_{1}+1}^{k} p_{i j k_{2}}^{0}+$ $\sum_{k_{3}=k_{1}}^{k-1} u\left(z_{i j k_{3}}^{0}, z_{i, j, k_{3}+1}^{0}\right)<e_{I(i j k)}^{0}$ and $s_{I(i j k)}^{0}>s_{i j k_{1}}+\sum_{k_{2}=k_{1}}^{k-1} p_{i j k_{2}}^{0}+\sum_{k_{3}=k_{1}}^{k-1} u\left(z_{i j k_{3}}^{0}, z_{i, j, k_{3}+1}^{0}\right)$. 
If the starting time of $o_{i j k_{1}}$ is not changed, the processing time of operation $o_{i j k_{1}^{*}}$ in $V_{1}^{*}$ is adjusted to $p_{i j k_{1}^{*}}^{\min }$, the earliest starting time of operation in $V_{2}^{*}$ is not changed, the processing time of operation $O_{i^{1} j^{1} k_{3}^{*}}$ in $V_{2}^{*}-\left\{O_{i^{1} j^{1} k^{1}}\right\}$ is adjusted to $p_{i^{1} j^{\prime} k_{3}^{*}}^{\max }$, and the processing time of operation $o_{i^{1} j^{1} k^{1}}$ is adjusted to $p_{i^{\prime} j^{\prime} k^{\prime}}^{\min }$, the processing conflict may be resolved. According to the formula (15), the new processing conflict time (denoted as $\left.\left(\chi_{I(i j k)}^{i j k}\right)^{\prime}\right)$ can be recalculated as follows $\left(k=k_{1}, \ldots, \theta_{i j}\right)$ :

$$
\begin{aligned}
& \left(\chi_{I(j i k)}^{i j k}\right)^{\prime} \\
& =\min \left(e_{i j k_{1}}+\sum_{k_{2}=k_{1}+1}^{k} p_{i j k_{2}}^{0}-\sum_{l_{1}^{*} \in V_{1}^{*}} \varepsilon_{l_{1}^{*}}+\sum_{k_{3}=k_{1}}^{k-1} u\left(z_{i j k_{3}}^{0}, z_{i, j, k_{3}+1}^{0}\right), e_{I(j i k)}^{0}+\sum_{l_{2}^{*} \in V_{2}^{*}\left\{\left\{o_{\left.i j^{\prime} k^{\prime}\right\}}\right.\right.} \varepsilon_{l_{2}^{*}}\right) \\
& -\max \left(s_{i j k_{1}}+\sum_{k_{2}=k_{1}}^{k-1} p_{i j k_{2}}^{0}-\sum_{l_{1}^{*} \in V_{1}^{*}\left\{\left\{o_{i j k}\right\}\right.} \varepsilon_{l_{1}^{*}}+\sum_{k_{3}=k_{1}}^{k-1} u\left(z_{i j k_{3}}^{0}, z_{i, j, k_{3}+1}^{0}\right), s_{I(i j k)}^{0}+\sum_{l_{2}^{*} \in V_{2}^{*}} \varepsilon_{l_{2}^{*}}\right)
\end{aligned}
$$

Because $e_{i j k_{1}}+\sum_{k_{2}=k_{1}+1}^{k} p_{i j k_{2}}^{0}+\sum_{k_{3}=k_{1}}^{k-1} u\left(z_{i j k_{3}}^{0}, z_{i, j, k_{3}+1}^{0}\right)<e_{I(i j k)}^{0}$ and $s_{I(i j k)}^{0}>s_{i j k_{1}}+$ $\sum_{k_{2}=k_{1}}^{k-1} p_{i j k_{2}}^{0}+\sum_{k_{3}=k_{1}}^{k-1} u\left(z_{i j k_{3}}^{0}, z_{i, j, k_{3}+1}^{0}\right), e_{i j k_{1}}+\sum_{k_{2}=k_{1}+1}^{k} p_{i j k_{2}}^{0}-\sum_{l_{1}^{*} \in V_{1}^{v_{1}}} \varepsilon_{l_{1}^{*}}+\sum_{k_{3}=k_{1}}^{k-1} u\left(z_{i j k_{3}}^{0}, z_{i, j, k_{3}+1}^{0}\right)<$ $e_{I(j i k)}^{0}+\sum_{l_{2}^{*} \in V_{2}^{*}\left\{o_{i i^{1} k^{\prime}}\right\}} \varepsilon_{l_{2}^{*}}$ and $s_{I(i j k)}^{0}+\sum_{l_{2}^{*} \in V_{2}^{*}} \varepsilon_{l_{2}^{*}}>s_{i j k_{1}}+\sum_{k_{2}=k_{1}}^{k-1} p_{i j k_{2}}^{0}-\sum_{l_{1}^{*} \in V_{1}^{*}\left\{o_{i j k}\right\}} \varepsilon_{l_{1}^{*}}+$ $\sum_{k_{3}=k_{1}}^{k-1} u\left(z_{i j k_{3}}^{0}, z_{i, j, k_{3}+1}^{0}\right)$. Then the formula (42) can be rewritten as follows:

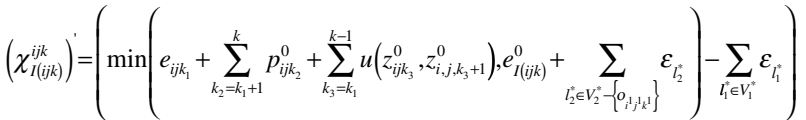

$$
\begin{aligned}
& -\left(\max \left(s_{i j k_{1}}+\sum_{k_{2}=k_{1}}^{k-1} p_{i j k_{2}}^{0}-\sum_{l_{1}^{*} \in V_{1}^{*} \backslash\left\{o_{i j k}\right\}} \varepsilon_{l_{1}^{*}}+\sum_{k_{3}=k_{1}}^{k-1} u\left(z_{i j k_{3}}^{0}, z_{i, j, k_{3}+1}^{0}\right), s_{I(j i k)}^{0}\right)+\sum_{l_{i} \in V_{2}^{*}} \varepsilon_{l_{2}^{*}}\right) \\
& =\min \left(e_{i j k_{1}}+\sum_{k_{2}=k_{1}+1}^{k} p_{i j k_{2}}^{0}+\sum_{k_{3}=k_{1}}^{k-1} u\left(z_{i j k_{3}}^{0}, z_{i, j, k_{3}+1}^{0}\right), e_{I(j i k)}^{0}+\sum_{l_{2}^{*} \in V_{2}^{*}\left\{f_{i, 1, k^{\prime}}\right\}} \varepsilon_{l_{2}^{k_{2}}}\right)
\end{aligned}
$$

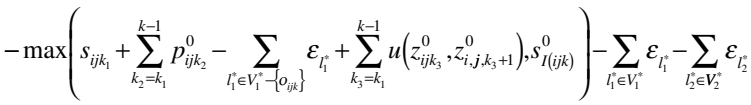

$$
\begin{aligned}
& =\min \left(e_{i j k_{1}}+\sum_{k_{2}=k_{1}+1}^{k} p_{i j k_{2}}^{0}+\sum_{k_{3}=k_{1}}^{k-1} u\left(z_{i j k_{3}}^{0}, z_{i, j, k_{3}+1}^{0}\right), e_{I(j i k)}^{0}\right) \\
& -\max \left(s_{i j k_{1}}+\sum_{k_{2}=k_{1}}^{k-1} p_{i j k_{2}}^{0}+\sum_{k_{3}=k_{1}}^{k-1} u\left(z_{i j k_{3}}^{0}, z_{i, j, k_{3}+1}^{0}\right), s_{I(j i k)}^{0}\right)-\sum_{l_{i} \in V_{1}^{*}} \varepsilon_{l_{1}^{*}}-\sum_{l_{2} \in V_{2}} \varepsilon_{l_{2}^{*}} \\
& =\chi_{I(j i k)}^{i j k}-\sum_{l_{i}^{\prime} \in V_{1}^{*}} \varepsilon_{l_{1}^{*}}-\sum_{l_{i}^{*} \in V_{2}^{*}} \varepsilon_{l_{2}^{*}}
\end{aligned}
$$

If $\sum_{l_{1}^{*} \in V_{1}^{*}} \varepsilon_{l_{1}^{*}}+\sum_{l_{2}^{*} \in V_{2}^{*}} \varepsilon_{l_{2}^{*}}-\chi_{I(i j k)}^{i j k} \geq 0$, then $\left(\chi_{I(i j k)}^{i j k}\right)^{\prime} \leq 0$. It can resolve the processing conflict between $o_{i j k}$ and $o_{I(i j k)}$ synchronously by adjusting the processing time of operations of $L_{i j}$ and charge $L_{i^{1} j^{1}}$. So, according to the classification of processing conflict of scheduling plan (section 2.3), the abnormal condition is three-level processing conflict $\zeta_{23}$.

- Case 2: $o_{i j k}$ is processed after $o_{I(i j k)}$ in the new scheduling plan. It can be known that $s_{i j k_{1}}+\sum_{k_{2}=k_{1}}^{k-1} p_{i j k_{2}}^{0}+\sum_{k_{3}=k_{1}}^{k-1} u\left(z_{i j k_{3}}^{0}, z_{i, j, k_{3}+1}^{0}\right)>s_{I(j i k)}^{0}$ and $e_{I(j i k)}^{0}<e_{i j k_{1}}+\sum_{k_{2}=k_{1}+1}^{k} p_{i j k_{2}}^{0}+\sum_{k_{3}=k_{1}}^{k-1} u\left(z_{i j k_{3}}^{0}, z_{i, j, k_{3}+1}^{0}\right)$. If the starting time of $o_{i j k_{1}}$ is not changed, the processing time of operation $o_{i j k_{4}^{*}}$ in $V_{1}^{*}-\left\{o_{i j k}\right\}$ is adjusted to $p_{i j k_{4}^{*}}^{\max }$, the processing time of oper- ation $o_{i j k}$ is adjusted to $p_{i j k}^{\min }$, the earliest starting time of operation in $V_{2}^{*}$ is not changed, the processing time of operation $o_{i^{1} j^{1} k_{2}^{*}}$ in $V_{2}^{*}$ is adjusted to $p_{i^{\prime} j^{1} k_{2}^{*}}^{\min }$, the processing conflict may be resolved. According to the formula (15), the new processing conflict time (denoted as $\left(\chi_{I(i j k)}^{i j k}\right)$ ) can be recalculated as follows $\left(k=k_{1}, \ldots, \theta_{i j}\right)$ :

$$
\begin{aligned}
& \left(\chi_{I(i j k)}^{i j k}\right)^{\prime} \\
& =\min \left(e_{i j k_{1}}+\sum_{k_{2}=k_{1}+1}^{k} p_{i j k_{2}}^{0}+\sum_{l_{1}^{*} \in V_{1}^{*}\left\{\left\{_{i j k}\right\}\right.} \varepsilon_{l_{1}^{*}}+\sum_{k_{3}=k_{1}}^{k-1} u\left(z_{i j k_{3}}^{0}, z_{i, j, k_{3}+1}^{0}\right), e_{I(i j k)}^{0}-\sum_{l_{2}^{*} \in V_{2}^{*}} \varepsilon_{l_{2}^{*}}\right) \\
& -\max \left(s_{i j k_{1}}+\sum_{k_{2}=k_{1}}^{k-1} p_{i j k_{2}}^{0}+\sum_{l_{1}^{*} \in V_{1}^{*}} \varepsilon_{l_{1}^{*}}+\sum_{k_{3}=k_{1}}^{k-1} u\left(z_{i j k_{3}}^{0}, z_{i, j, k_{3}+1}^{0}\right), s_{I(i j k)}^{0}-\sum_{l_{2}^{*} \in V_{2}^{*}\left\{\left\{_{i_{i} j_{k} k^{\prime}}\right\}\right.} \varepsilon_{l_{2}^{*}}\right)
\end{aligned}
$$

Because $s_{i j k_{1}}+\sum_{k_{2}=k_{1}}^{k-1} p_{i j k_{2}}^{0}+\sum_{k_{3}=k_{1}}^{k-1} u\left(z_{i j k_{3}}^{0}, z_{i, j, k_{3}+1}^{0}\right)>s_{I(i j k)}^{0}$ and $e_{I(j i k)}^{0}<$ $e_{i j k_{1}}+\sum_{k_{2}=k_{1}+1}^{k} p_{i j k_{2}}^{0}+\sum_{k_{3}=k_{1}}^{k_{2}=k_{1}} u\left(z_{i j k_{3}}^{0}, z_{i, j, k_{3}+1}^{0}\right), \quad s_{i j k_{1}}+\sum_{k_{2}=k_{1}}^{k-1} p_{i j k_{2}}^{0}+\sum_{l_{1}^{*} \in V_{1}^{*}} \varepsilon_{l_{1}^{*}}+$ $\sum_{k_{3}=k_{1}}^{k-1} u\left(z_{i j k_{3}}^{0}, z_{i, j, k_{3}+1}^{0}\right)>s_{I(i j k)}^{0}-\sum_{l_{2}^{*} \in V_{2}^{*}-\left\{o_{i, j j_{k} k^{1}}\right\}} \varepsilon_{l_{2}^{*}}$ and $e_{I(j i k)}^{0}-\sum_{l_{2}^{*} \in V_{2}^{*}} \varepsilon_{l_{2}^{*}}<e_{i j k_{1}}+$ $\sum_{k_{2}=k_{1}+1}^{k} p_{i j k_{2}}^{0}+\sum_{l_{1}^{*} \in V_{1}^{*}\left\{0_{i j k}\right\}} \varepsilon_{l_{1}^{*}}+\sum_{k_{3}=k_{1}}^{k-1} u\left(z_{i j k_{3}}^{0}, z_{i, j, k_{3}+1}^{0}\right)$. Then the formula (44) can be rewritten as follows:

$$
\begin{aligned}
& \left(\chi_{I(j i k)}^{i j k}\right)^{\prime}=\left(\min \left(e_{i j k_{1}}+\sum_{k_{2}=k_{1}+1}^{k} p_{i j k_{2}}^{0}+\sum_{l_{1}^{*} \in V_{1}^{*} \backslash\left\{o_{i j k}\right\}} \varepsilon_{l_{1}^{*}}+\sum_{k_{3}=k_{1}}^{k-1} u\left(z_{i j k_{3}}^{0}, z_{i, j, k_{3}+1}^{0}\right), e_{I(j i k)}^{0}\right)-\sum_{l_{2} \in V_{2}^{*}} \varepsilon_{l_{2}^{*}}\right) \\
& -\left(\max \left(s_{i j k_{1}}+\sum_{k_{2}=k_{1}}^{k-1} p_{i j k_{2}}^{0}+\sum_{k_{3}=k_{1}}^{k-1} u\left(z_{i j k_{3}}^{0}, z_{i, j, k_{3}+1}^{0}\right), s_{I(j i k)}^{0}-\sum_{l_{2} \in V_{2}^{*}\left\{o_{i, j}\right\}} \varepsilon_{l^{\prime}}\right)+\sum_{l_{i} \in V_{1}^{*}} \varepsilon_{l_{1}^{\prime}}\right) \\
& =\min \left(e_{i j k_{1}}+\sum_{k_{2}=k_{1}+1}^{k} p_{i j k_{2}}^{0}+\sum_{\left.\left.l_{i} \in V_{1}^{*}\right\} \rho_{0 j k}\right\}} \varepsilon_{l_{l}^{*}}+\sum_{k_{3}=k_{1}}^{k-1} u\left(z_{i j k_{3}}^{0}, z_{i, j, k_{3}+1}^{0}\right), e_{I(j i k)}^{0}\right)
\end{aligned}
$$

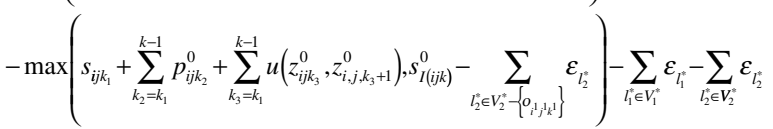

$$
\begin{aligned}
& =\min \left(e_{i j k_{1}}+\sum_{k_{2}=k_{1}+1}^{k} p_{i j k_{2}}^{0}+\sum_{k_{3}=k_{1}}^{k-1} u\left(z_{i j k_{3}}^{0}, z_{i, j, k_{3}+1}^{0}\right), e_{I(j i k)}^{0}\right) \\
& -\max \left(s_{i j k_{1}}+\sum_{k_{2}=k_{1}}^{k-1} p_{i j k_{2}}^{0}+\sum_{k_{3}=k_{1}}^{k-1} u\left(z_{i j k_{3}}^{0}, z_{i, j, k_{3}+1}^{0}\right), s_{I(j i k)}^{0}\right)-\sum_{l_{1} \in V_{1}^{*}} \varepsilon_{l_{1}^{\prime \prime}}-\sum_{l_{i}^{\prime} \in V_{2}^{*}} \varepsilon_{l_{2}^{*}} \\
& =\chi_{I(j i k)}^{i j k}-\sum_{l_{1} \in V_{1}^{*}} \varepsilon_{l_{1}^{*}}-\sum_{l_{2} \in V_{2}^{*}} \varepsilon_{l_{2}^{*}}
\end{aligned}
$$

If $\sum_{l_{1}^{*} \in V_{1}^{*}} \varepsilon_{l_{1}^{*}}+\sum_{l_{2}^{*} \in V_{2}^{*}} \varepsilon_{l_{2}^{*}}-\chi_{I(i j k)}^{i j k} \geq 0$, then $\left(\chi_{I(j i k)}^{i j k}\right)^{\prime} \leq 0$. It can resolve the processing conflict between $o_{i j k}$ and $o_{I(i j k)}$ synchronously by adjusting the processing time of operations of $L_{i j}$ and charge $L_{i^{1}{ }^{1}{ }^{1}}$. So, according to the classification of processing conflict of scheduling plan (section 2.3), the abnormal condition is three-level processing conflict $\zeta_{23}$.

To sum up, if $\varepsilon_{l_{1}}+\varepsilon_{l_{2}}-\chi_{I(i j k)}^{i j k}<0$, then let $\varepsilon_{l}=0$ for the nodes $l \mid l \in V, l \notin V_{1}, l \notin V_{2}$. If $\sum_{l_{1}^{*} \in V_{1}^{*}} \varepsilon_{l_{1}^{*}}+\sum_{l_{2}^{*} \in V_{2}^{*}} \varepsilon_{l_{2}^{*}}-\chi_{I(i j k)}^{i j k} \geq 0$, then the abnormal condition of scheduling plan is three-level processing conflict $\zeta_{23}$.

(5) If $\sum_{l_{1}^{*} \in V_{1}^{*}} \varepsilon_{l_{1}^{*}}+\sum_{l_{2}^{*} \in V_{2}^{*}} \varepsilon_{l_{2}^{*}}-\chi_{I(j i k)}^{i j k}<0$ when $\varepsilon_{l}=0$ for the nodes $l \mid l \in V, l \notin V_{1}, l \notin V_{2}$ and $\tilde{\chi}_{I(i j k)}^{i j k} \leq 0$ for the nodes $l \mid l \in V$, then the abnormal condition of scheduling plan is four-level processing conflict $\zeta_{24}$. 
Proof. According to above analysis, it can not resolve the processing conflict between $o_{i j k}$ and $o_{I(i j k)}$ synchronously by adjusting the processing time of operations of $L_{i j}$ and the charge including $o_{I(i j k)}$ if $\sum_{l_{1}^{*} \in V_{1}^{*}} \varepsilon_{l_{1}^{*}}+\sum_{l_{2}^{*} \in V_{2}^{*}} \varepsilon_{l_{2}^{*}}-\chi_{I(j i k)}^{i j k}<0$. The processing conflict may be resolved synchronously by adjusting the processing time of operations of $L_{i j}$, the charge including $o_{I(i j k)}$ and other charges. Section 4.3.4 analyzes the new processing conflict time in detail when buffer units are considered and the new processing conflict time $\tilde{\chi}_{I(i j k)}^{i j k}$ is obtained according to the formula (26). Moreover, according to the analysis of formula (26) in section 4.3.4, it can resolve the processing conflict between $o_{i j k}$ and $o_{I(i j k)}$ synchronously by adjusting the processing time of operations of $L_{i j}$, charge including $o_{I(i j k)}$ and other charges if $\tilde{\chi}_{I(i j k)}^{i j k} \leq 0$ when $\varepsilon_{l} \neq 0$ for the nodes $l \mid l \in V$. So, the abnormal condition is four-level processing conflict $\zeta_{24}$ according to the classification of processing conflict of scheduling plan (section 2.3).

(6) For the nodes $l \mid l \in V$, if $\tilde{\chi}_{I(j i k)}^{i j k}>0$, then the abnormal condition of scheduling plan is five-level processing conflict $\zeta_{25}$.

Proof. According to above analysis, if $\tilde{\chi}_{I(j i k)}^{i j k}>0$ when $\varepsilon_{l} \neq$ 0 for the nodes $l \mid l \in V$, it can not resolve the processing conflict between $o_{i j k}$ and $o_{I(i j k)}$ only by adjusting the processing time of operations of $L_{i j}$, charge including $o_{I(i j k)}$ and other charges. Because $\tilde{\chi}_{I(i j k)}^{i j k}>0$ for the nodes $l \mid l \in V$, the original buffer capacity can not satisfy the demand for resolving the processing conflict. The only way is to increase the buffer capacity which can affect the $o_{i j k}$ and $o_{I(i j k)}$. It can change the buffer units which affect the $o_{i j k}$ and $o_{I(i j k)}$ by adjusting the processing machines of operations at steelmaking stage and refining stage. Then it may resolve the processing conflict between $o_{i j k}$ and $o_{I(i j k)}$ by the new buffer units, namely adjusting the processing time of operations at each stage. We can obtained the new processing conflict time (denoted as $\left(\tilde{\chi}_{I(i j k)}^{i j k}\right)$ ) according to the formula (26) when the new buffer units are considered. If $\left(\tilde{\chi}_{I(i j k)}^{i j k}\right)^{\prime} \leq 0$, the processing conflict can be resolved by the new buffer units. So, according to the classification of the processing conflict of scheduling plan (section 2.3), the abnormal condition is five-level the processing conflict $\zeta_{25}$.

\section{Industrial Application}

\subsection{Application Background}

The Baosteel factory of China can produce 1000 kinds steel grade. There are three parallel converters of $250 \mathrm{t}$ at steel making stage (4LD, 5LD, 6LD), three kinds of refining machines (5RH-1, 5RH-2, 3RH, LF-1, LF-2, IR_UT) at the refining stage, and three continuous casters $(\overline{4} \mathrm{CC}, 5 \mathrm{CC}$, $6 \mathrm{CC})$. The number of refining processing ranging is from 1 to 4 , and refining routes are more than 20 . The computer systems have the level 2 computer system of process control and the level 3 computer system of region management. In the SMCC production process, operation time delay often occurs which may lead to planned casting break or processing conflict so that the initial scheduling plan becomes unrealizable. The rescheduling for the operation time delay is mainly relies on manual adjustment. The rescheduling methods are simply divided into four categories by the artificial experience: (1) if the operation time delay is within five minutes, then the scheduling plan is not adjusted. (2) If the operation time delay is between five minutes and ten minutes, then adjusting the starting time and the completion time of all operations. (3) If the operation time delay is between ten minutes and thirty minutes, then adjusting the processing machine, the starting time and the completion time of all operations. (4) If the operation time delay is greater than thirty minutes, then using other methods. Existing manual rescheduling methods with disturbances don't analyze the influence degree of disturbances to the initial scheduling plan in detail, so the adjustment degree of initial scheduling plan is always too greater, which leads to the poor continuity and stability of initial scheduling plan.

\subsection{Application Example}

This paper takes the actual rescheduling problem in BaoSteel plant for example. Figure 3 shows the scheduling plan information at time $t_{l}$. The cast one is processed on $4 C C$ and $\Omega_{1}=\left\{L_{11}, L_{12}, L_{13}, L_{14}, L_{15}, L_{16}, L_{17}\right\}$. The cast two is processed on $5 \mathrm{CC}$ and $\Omega_{2}=\left\{L_{21}, L_{22}, L_{23}, L_{24}, L_{25}, L_{26}, L_{27}\right.$, $\left.L_{28}\right\}$. The cast three is processed on $6 \mathrm{CC}$ and $\Omega_{3}=\left\{L_{31}, L_{32}, L_{33}, L_{34}, L_{35}, L_{36}\right\}$. The standard processing time and processing time range are as shown in Table 1.

The processing time of charge on continuous casting machine is related to following facts: the total weight of the molten steel in charge, the average thickness of the slab, the average width of the odd strand slab and the dual slab of charge and pulling speed of the continuous casting machine. If the charge $L_{i j}$ is the first chare in the cast, then the processing time of the charge $L_{i j}$ on continuous casting machine is calculated as follows:

$$
p_{i, j, \theta_{i j}}=\frac{\omega_{i j} \times 10^{6}}{\alpha_{i j} \times \frac{\zeta_{i j}^{1}+\zeta_{i j}^{2}}{2} \times 7.8 \times 2 \times\left(v_{i j}-0.2\right)}
$$

where $\omega_{i j}$ is the total weight of the molten steel in charge $L_{i j}$, $\alpha_{i j}$ is the average thickness of the slab of charge $L_{i j}$ and $v_{i j}$ is the pulling speed. The $\zeta_{i j}^{1}$ and $\zeta_{i j}^{2}$ are the average width of the odd strand slab and the dual slab of charge $L_{i j}$, respectively. If the charge $L_{i j}$ is not the first chare in the cast, then the processing time of the charge $L_{i j}$ on continuous casting machine is calculated as follows:

$$
\begin{aligned}
p_{i, j, \theta_{i j}}= & \frac{\omega_{i j} \times 10^{6}-\gamma_{i j} \times 31.2 \times\left(\xi_{i j}^{1}+\xi_{i j}^{2}+\xi_{i, j-1}^{1}+\xi_{i, j-1}^{2}\right)}{\alpha_{i j} \times \frac{\left(\xi_{i j}^{1}+\xi_{i j}^{2}+\xi_{i, j-1}^{1}+\xi_{i, j-1}^{2}\right)}{4} \times 7.8 \times 2 \times\left(v_{i j}-0.2\right)} \\
& +\frac{8}{v_{i j}-0.2}
\end{aligned}
$$

where $\omega_{i j}$ is the total weight of the molten steel in charge $L_{i j}$, $\alpha_{i j}$ is the average thickness of the slab of charge $L_{i j}$ and $v_{i j}$ is the pulling speed. The $\zeta_{i j}^{1}$ and $\zeta_{i j}^{2}$ are the average width of the odd strand slab and the dual slab of charge $L_{i j}$, respectively. The $\zeta_{i, j-1}^{1}$ and $\zeta_{i, j-1}^{2}$ are the average width of the odd strand slab and the dual slab of charge $L_{i, j-1}$, respectively. The pulling speed includes the minimum pulling speed, standard pulling speed, the maximum pulling speed. The processing time of charges on continuous casting machine is shown in Tables $\mathbf{2}$ and $\mathbf{3}$ according to the formula (46) and formula (47). The transportation time is as shown in Table 4.

The scheduling plan at time $t_{2}\left(t_{2}=14: 21\right)$ is shown in Fig. 4. The operation $o_{241}$ on $5 \mathrm{LD}$ is starting at time $14: 21$, that is $s_{241}^{*}=14: 21$. The abnormal condition prediction of the scheduling plan is made as follows according to the pro-

Table 1. The processing time of operations at steelmaking and refining stage.

\begin{tabular}{c|c|c|c|c|c|c|c|c}
\hline \multirow{2}{*}{ Machine } & \multicolumn{2}{|c|}{ LD } & \multicolumn{2}{c|}{ RH } & \multicolumn{2}{c|}{ LF } & \multicolumn{2}{c}{ IR_UT } \\
\cline { 2 - 9 } & Standard & Interval & Standard & Interval & Standard & Interval & Standard & Interval \\
\hline $\begin{array}{c}\text { Processing } \\
\text { time (m) }\end{array}$ & 35 & {$[32,38]$} & 20 & {$[15,30]$} & 30 & {$[22,35]$} & 30 & {$[25,35]$} \\
\hline
\end{tabular}


posed method:

(1) Get event information: $m=2 L D, o=o_{241}, \chi=1$. $\tau=s_{241}^{*}-s_{241}^{0}=20>0$ because $s_{241}^{0}=14: 01$ and $s_{241}^{*}=14$ : 21 , so $y=y_{1}$, and $Y=\left\{o_{241}, 2 L D, y_{1}, 20\right\}$.

(2) The processing time of operations which are being processed or have been processed can not be changed. So, the operations not be processed are only considered when the reachability matrix is established. The correspondence relationship between nodes and operations is shows in Table 5. The $\kappa$ denotes the idle state, and the $\mu$ indicates the interval state between adjacent operations of the same charge.

Table 2. The processing time of charges at casting stage.

\begin{tabular}{l|ccccccccccc}
\hline \multicolumn{1}{c|}{ Operations } & $o_{113}$ & $o_{123}$ & $o_{133}$ & $o_{143}$ & $o_{153}$ & $o_{163}$ & $o_{173}$ & $o_{213}$ & $o_{223}$ & $o_{233}$ & $o_{243}$ \\
\hline $\begin{array}{l}\text { Minimal processing } \\
\text { time (m) }\end{array}$ & 45 & 45 & 45 & 50 & 55 & 45 & 40 & 45 & 45 & 50 & 45 \\
$\begin{array}{l}\text { Standard processing } \\
\text { time (m) }\end{array}$ & 48 & 48 & 49 & 58 & 60 & 46 & 42 & 46 & 46 & 50 & 50 \\
$\begin{array}{l}\text { Maximum processing } \\
\text { time (m) }\end{array}$ & 60 & 60 & 61 & 70 & 75 & 60 & 60 & 60 & 60 & 65 & 65 \\
\hline
\end{tabular}

Table 3. The processing time of charges at casting stage

\begin{tabular}{l|cccccccccc}
\hline \multicolumn{1}{c|}{ Operations } & $o_{253}$ & $o_{263}$ & $o_{273}$ & $o_{283}$ & $o_{313}$ & $o_{323}$ & $o_{333}$ & $o_{343}$ & $o_{353}$ & $o_{363}$ \\
\hline $\begin{array}{l}\text { Minimal processing } \\
\text { time (m) }\end{array}$ & 45 & 50 & 45 & 45 & 60 & 60 & 60 & 60 & 60 & 60 \\
$\begin{array}{l}\text { Standard processing } \\
\text { time (m) }\end{array}$ & 50 & 55 & 52 & 54 & 74 & 74 & 76 & 76 & 76 & 77 \\
$\begin{array}{l}\text { Maximum processing } \\
\text { time (m) }\end{array}$ & 65 & 70 & 67 & 70 & 85 & 85 & 85 & 85 & 85 & 85 \\
\hline
\end{tabular}

Table 4. The transportation time between machines.

\begin{tabular}{|c|c|c|c|c|c|c|c|c|c|c|c|c|}
\hline $\begin{array}{c}\text { Transportation } \\
\text { time }\end{array}$ & $4 \mathrm{LD}$ & $5 \mathrm{LD}$ & $6 \mathrm{LD}$ & $5 \mathrm{RH}-1$ & $5 \mathrm{RH}-2$ & $3 \mathrm{RH}$ & LF-1 & LF-2 & R_UT & $4 \mathrm{CC}$ & $5 \mathrm{CC}$ & $6 \mathrm{CC}$ \\
\hline $4 \mathrm{LD}$ & 0 & 12 & 13 & 10 & 10 & 13 & 15 & 15 & 13 & 16 & 15 & 14 \\
\hline $5 \mathrm{LD}$ & & 0 & 12 & 15 & 15 & 13 & 15 & 15 & 13 & 16 & 15 & 14 \\
\hline 6LD & & & 0 & 15 & 15 & 13 & 15 & 15 & 13 & 16 & 16 & 15 \\
\hline $5 \mathrm{RH}-1$ & & & & 0 & 18 & 21 & 20 & 18 & 23 & 22 & 25 & 25 \\
\hline $5 \mathrm{RH}-2$ & & & & & 0 & 21 & 20 & 18 & 23 & 22 & 25 & 25 \\
\hline $3 \mathrm{RH}$ & & & & & & 0 & 20 & 21 & 18 & 25 & 22 & 22 \\
\hline LF-1 & & & & & & & 0 & 18 & 21 & 22 & 25 & 25 \\
\hline LF-2 & & & & & & & & 0 & 23 & 22 & 25 & 25 \\
\hline IR_UT & & & & & & & & & 0 & 25 & 22 & 22 \\
\hline $4 \mathrm{CC}$ & & & & & & & & & & 0 & 16 & 16 \\
\hline $5 \mathrm{CC}$ & & & & & & & & & & & 0 & 13 \\
\hline $6 \mathrm{CC}$ & & & & & & & & & & & & 0 \\
\hline
\end{tabular}

(3) It can be known that the $o_{241}$ is the corresponding operation for the node 6 according to the Table 5 . The planned casting break time $\gamma_{3,4}^{2}$ between charge $L_{23}$ and charge $L_{24}$ is calculated as follows according to the formula (18): $\gamma_{3,4}^{2}=e_{241}^{0}+\Delta t+p_{242}^{0}+u\left(z_{241}^{0} z_{242}^{0}\right)+u\left(z_{242}^{0}, z_{2423}^{0}\right)-e_{233}^{0}=20$. Because $\gamma_{3,4}^{2}>0$, the planned casting break between $L_{23}$ and $L_{24}$ occurs. Because $p_{233}^{\max }-p_{233}^{0}-\gamma_{3,4}^{2}=65-20-20=$ $-5<0$, let $\varepsilon_{l}=0 \mid l \notin V_{24}$, then the planned casting break time $\tilde{\gamma}_{3,4}^{2}$ between $L_{23}$ and $L_{24}$ is calculated as follows according to the formula (27): $\tilde{\gamma}_{3,4}^{2}=\eta_{76}-\lambda_{75}=12>0$ and $p_{233}^{\max }-p_{233}^{0}-\tilde{\gamma}_{3,4}^{2}=3>0$, so the abnormal condition of planned casting break is two-level planned casting break $\zeta_{12}$.

(4) The processing conflict time $\chi_{251}^{241}$ of charge $L_{24}$ on 5LD is calculated as follows according to the formula (15): $\chi_{251}^{241}=\min \left(e_{241}^{0}+\Delta t, e_{251}^{0}\right)-\max \left(s_{241}^{0}+\Delta t, s_{251}^{0}\right)=5>0$, so

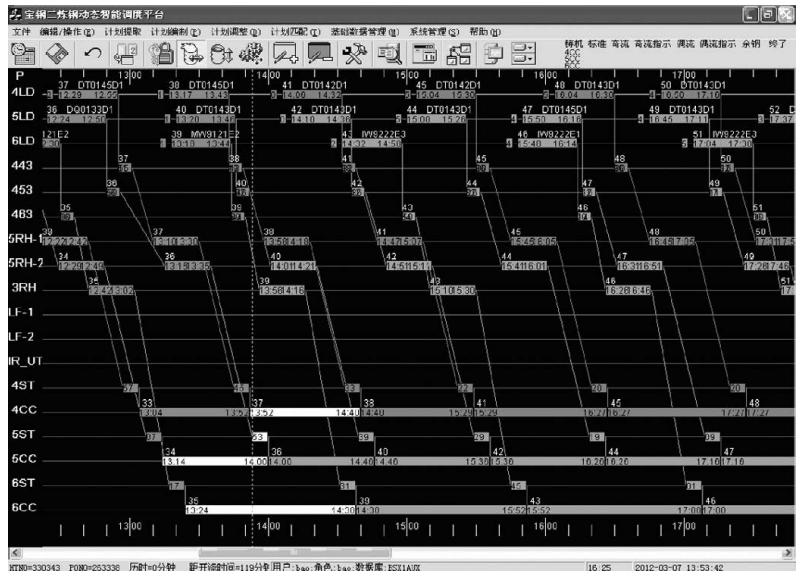

Fig. 3. The scheduling plan at time $t_{1}$.

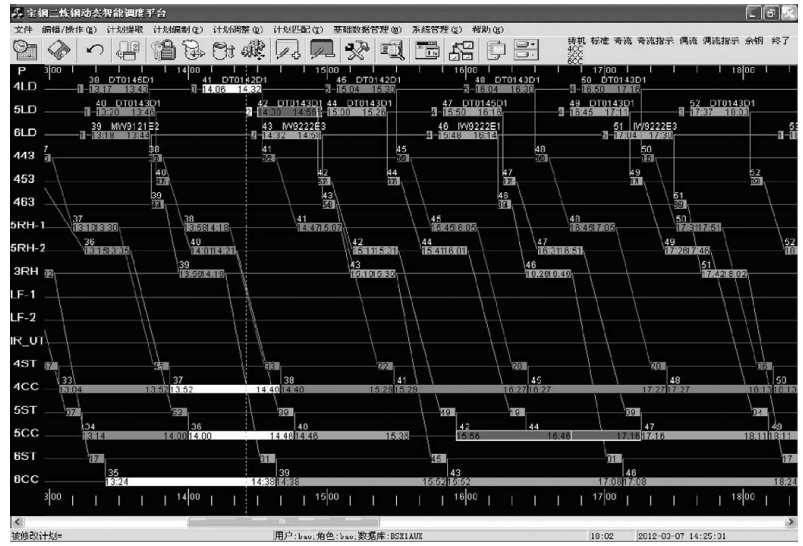

Fig. 4. The scheduling plan at time $t_{2}$.

Table 5. The correspondence relationship between nodes and operations.

\begin{tabular}{c|ccccccccccccccccccccc}
\hline Node & 1 & 2 & 3 & 4 & 5 & 6 & 7 & 8 & 9 & 10 & 11 & 12 & 13 & 14 & 15 & 16 & 17 & 18 & 19 & 20 \\
\hline Operation & $o_{151}$ & $\kappa_{161}^{151}$ & $o_{161}$ & $\kappa_{171}^{161}$ & $o_{171}$ & $o_{241}$ & $\kappa_{251}^{241}$ & $o_{251}$ & $\kappa_{261}^{251}$ & $o_{261}$ & $\kappa_{271}^{261}$ & $o_{271}$ & $\kappa_{281}^{271}$ & $o_{281}$ & $o_{331}$ & $\kappa_{341}^{331}$ & $o_{341}$ & $\kappa_{351}^{341}$ & $o_{351}$ & $\kappa_{361}^{351}$ \\
\hline Node & 21 & 22 & 23 & 24 & 25 & 26 & 27 & 28 & 29 & 30 & 31 & 32 & 33 & 34 & 35 & 36 & 37 & 38 & 39 & 40 \\
\hline Operation & $o_{361}$ & $\mu_{152}^{151}$ & $\mu_{162}^{161}$ & $\mu_{172}^{171}$ & $\mu_{242}^{241}$ & $\mu_{252}^{251}$ & $\mu_{262}^{261}$ & $\mu_{272}^{271}$ & $\mu_{282}^{281}$ & $\mu_{332}^{331}$ & $\mu_{342}^{341}$ & $\mu_{352}^{351}$ & $\mu_{362}^{361}$ & $o_{142}$ & $\kappa_{152}^{142}$ & $o_{152}$ & $\kappa_{162}^{152}$ & $o_{162}$ & $\kappa_{172}^{162}$ & $o_{172}$ \\
\hline Node & 41 & 42 & 43 & 44 & 45 & 46 & 47 & 48 & 49 & 50 & 51 & 52 & 53 & 54 & 55 & 56 & 57 & 58 & 59 & 60 \\
\hline Operation & $o_{242}$ & $\kappa_{252}^{242}$ & $o_{252}$ & $\kappa_{262}^{252}$ & $o_{262}$ & $\kappa_{272}^{262}$ & $o_{272}$ & $\kappa_{282}^{272}$ & $o_{282}$ & $o_{332}$ & $\kappa_{342}^{332}$ & $o_{342}$ & $\kappa_{352}^{342}$ & $o_{352}$ & $\kappa_{362}^{352}$ & $o_{362}$ & $\mu_{143}^{142}$ & $\mu_{153}^{152}$ & $\mu_{163}^{162}$ & $\mu_{173}^{172}$ \\
\hline Node & 61 & 62 & 63 & 64 & 65 & 66 & 67 & 68 & 69 & 70 & 71 & 72 & 73 & 74 & 75 & 76 & 77 & 78 & 79 & 80 \\
\hline Operation & $\mu_{243}^{242}$ & $\mu_{253}^{252}$ & $\mu_{263}^{262}$ & $\mu_{273}^{272}$ & $\mu_{283}^{282}$ & $\mu_{333}^{332}$ & $\mu_{343}^{342}$ & $\mu_{353}^{352}$ & $\mu_{363}^{362}$ & $o_{133}$ & $o_{143}$ & $o_{153}$ & $o_{163}$ & $o_{173}$ & $o_{233}$ & $o_{243}$ & $o_{253}$ & $o_{263}$ & $o_{273}$ & $o_{283}$ \\
\hline Node & 81 & 82 & 83 & 84 & 85 & & & & & & & & & & \\
\hline Operation & $o_{323}$ & $o_{333}$ & $o_{343}$ & $o_{353}$ & $o_{363}$ & & & & & & & & & \\
\hline
\end{tabular}


the processing conflict between $L_{24}$ and $L_{25}$ on 5LD occurs. Because $\varepsilon_{6}-\chi_{251}^{241}=-2<0, \quad \varepsilon_{8}-\chi_{251}^{241}=-2<0$ and $\varepsilon_{6}+$ $\varepsilon_{8}-\chi_{251}^{241}=1>0$, then the abnormal condition of processing conflict is two-level processing conflict $\zeta_{22}$.

(5) The processing conflict time $\chi_{252}^{242}$ is calculated as follows according to the formula (15): $\chi_{252}^{242}=\min \left(e_{241}^{0}+\Delta t+\right.$ $\left.u\left(z_{241}^{0}, z_{242}^{0}\right)+p_{242}^{0}, e_{252}^{0}\right)-\max \left(e_{241}^{0}+\Delta t+u\left(z_{241}^{0}, z_{242}^{0}\right), s_{252}^{0}\right)=$ $-10<0$, so there is no processing conflict between $L_{24}$ and $L_{25}$ on $5 \mathrm{RH}-2$.

(6) The processing conflict time $\chi_{253}^{243}$ is calculated as follows according to the formula (15): $\chi_{253}^{243}=\min \left(e_{241}^{0}+\Delta t+\right.$ $\left.u\left(z_{241}^{0}, z_{242}^{0}\right)+p_{242}^{0}+u\left(z_{242}^{0}, z_{243}^{0}\right)+p_{243}^{0}, e_{253}^{0}\right)-\max \left(e_{241}^{0}+\Delta t+\right.$ $\left.u\left(z_{241}^{0}, z_{242}^{0}\right)+p_{242}^{0}+u\left(z_{242}^{0}, z_{243}^{0}\right), s_{253}^{0}\right)=20>0$, so the processing conflict between $L_{24}$ and $L_{25}$ on $5 \mathrm{CC}$ occurs. $\varepsilon_{76}-\chi_{253}^{243}=-15<0, \quad \varepsilon_{77}-\chi_{251}^{241}=-15<0$ and $\varepsilon_{76}+\varepsilon_{77}-$ $\chi_{253}^{243}=-10<0$. When let $\varepsilon_{l}=0\left|l \notin V_{24},\right| l \notin V_{25}, \tilde{\chi}_{253}^{243}=-1<$ 0 , so the abnormal condition of processing conflict is threelevel processing conflict $\zeta_{23}$.

\subsection{Application Effect}

According to the above application example, it can be known that abnormal condition of scheduling plan with operation time delay disturbance are determined according the proposed method when the operation delay time of $o_{241}$ is twenty minutes. (1) The abnormal condition of planned casting break between the charge $L_{23}$ and the charge $L_{24}$ on $5 \mathrm{CC}$ is two-level planned casting break $\zeta_{12}$, so the planned casting break between charge can be resolved synchronously by adjusting the processing time of $L_{23}$ at continuous casting stage and the processing time of $L_{24}$ at each stage. (2) The abnormal condition of processing conflict between the charge $L_{24}$ and the charge $L_{25}$ on 5LD is two-level processing conflict $\zeta_{22}$, so the processing conflict between charge $L_{24}$ and $L_{25}$ can be resolved synchronously by adjusting the processing time of $L_{24}$ and $L_{25}$ on 5LD. (3) The abnormal condition of processing conflict between the charge $L_{24}$ and the charge $L_{25}$ on $5 \mathrm{CC}$ is three-level processing conflict $\zeta_{23}$, so the processing conflict between charge $L_{24}$ and $L_{25}$ can be resolved synchronously by adjusting the processing time of $L_{24}$ and $L_{25}$ on each stage. Because the operation delay time of $o_{241}$ is twenty minutes which is between ten minutes and thirty minutes, the abnormal condition of the scheduling plan will be resolved by adjusting the processing machine, the starting time and the completion time of all operations according to the manual method. The proposed method can solve the abnormal condition only by adjusting the processing time of some charges, which can maintain the good continuity and stability.

\section{Conclusions}

In the steelmaking and continuous casting (SMCC) production process, operation time delay often occurs which may lead to planned casting break or processing conflict so that the initial scheduling plan becomes unrealizable. Existing rescheduling methods don't analyze the influence degree of disturbances to the initial scheduling plan in detail, so the adjustment degree of initial scheduling plan is always too greater, which leads to the poor continuity and stability of initial scheduling plan. In this paper, the relation among operation time delay, planned casting break and processing conflict is firstly deeply analyzed. Then a novel prediction method for abnormal condition of scheduling plan with operation time delay disturbance in SMCC production process is proposed including disturbance identification of operating time delay based on event-driven mechanism, analysis on charges based on reachability matrix, analysis on influence degree of disturbance and abnormal condition decision of initial scheduling plan. The real-time application shows that the proposed prediction method can timely and accurately predict the abnormal condition of the scheduling plan with operation time delay disturbance, which can only adjust the affected charges that must to be rescheduled in the initial scheduling plan and reduce the frequency of complete rescheduling. The initial scheduling plan can also maintain the good continuity and stability.

\section{Acknowledgements}

This research is partly supported by National Basic Research Program of China (2009CB320601), National Natural Science Foundation of China (61104174, 61174187); 111 Project (No. B08015), Basic Scientific Research Foundation of Northeast University under Grant N110208001, Starting Foundation of Northeast University under Grant 29321006.

\section{REFERENCES}

1) E. Sanmarti, A. Espuna and L. Puigianer: Comput. Chem. Eng., 21 (1997), 1157.

2) G. E. Vieira, J. W. Herrmann and E. Lin: Int. J. Prod. Res., 38 (2000), 1899.

3) R. J. Abumaizar and J. A. Svestka: Int. J. Prod. Res., 35 (1997), 2065.

4) D. Pacciarelli and M. Pranzo: Comput. Chem. Eng., 28 (2004), 2832.

5) H. Missbaucer, W. Hauber and W. Stadler: Int. J. Prod. Res., 47 (2009), 4147.

6) A. Atighehchian, M. Bijari and H. Tarkesh: Comput. Oper. Res., 36 (2009), 2450.

7) R. Roy, B. A. Adesola and S. Thornton: Int. J. Prod. Res., 42 (2004), 3975.

8) D. F. Guo and T. K. Li: 2007 2nd IEEE Conf. on Industrial Electronics and Applications, IEEE, Piscataway, NJ, (2007), 1421.

9) T. K. Li and D. F. Guo: Lecture Notes in Computer Science, Springer, Berlin, (2007), 1108.

10) C. S. Zhang, T. K. Li and B. L. Wang: Energy Procedia, 13 (2011), 253.

11) K. Chen, Z. Zheng and Y. Liu: IEEE International Conf. on Industrial Engineering and Engineering Management, IEEE, Piscataway, NJ, (2010), 2366.

12) P. Cowling and M. Johansson: Eur. J. Oper. Res., 139 (2002), 230. 